Bull. Soc. math. France

130 (1), 2002, p. 1-33

\title{
RÉSULTATS SUR LA CONJECTURE DE DUALITÉ ÉTRANGE SUR LE PLAN PROJECTIF
}

\author{
par Gentiana Danila
}

\begin{abstract}
RÉsumÉ. - La conjecture de «dualité étrange » de Le Potier donne un isomorphisme entre l'espace des sections du fibré déterminant sur deux espaces de modules différents de faisceaux semi-stables sur le plan projectif $\mathbb{P}_{2}$. On considère deux classes orthogonales $c, u$ dans l'algèbre de Grothendieck $\mathrm{K}\left(\mathbb{P}_{2}\right)$ telles que $c$ est de rang strictement positif et $u$ est de rang zéro, et on note $\mathrm{M}_{c}$ et $\mathrm{M}_{u}$ les espaces de modules de faisceaux semi-stables de classe $c$, respectivement $u$ sur $\mathbb{P}_{2}$. Il existe sur $\mathrm{M}_{c}\left(\right.$ resp. $\left.\mathrm{M}_{u}\right)$ un fibré déterminant inversible $\mathcal{D}_{u}$ (resp. $\mathcal{D}_{c}$ ) et le produit tensoriel externe $\mathcal{D}_{c} \otimes \mathcal{D}_{c}$ sur l'espace produit $\mathrm{M}_{c} \otimes \mathrm{M}_{c}$ a une section canonique $\sigma_{c, u}$ qui fournit une application linéaire $\mathcal{D}_{c, u}: \mathrm{H}^{0}\left(\mathrm{M}_{u}, \mathcal{D}_{c}\right)^{*} \rightarrow \mathrm{H}^{0}\left(\mathrm{M}_{c}, \mathcal{D}_{u}\right)$. Si $\mathrm{M}_{c}$ n'est pas vide, la conjecture affirme que $\mathcal{D}_{c, u}$ est un isomorphisme. Nous prouvons la conjecture dans le cas particulier où $c$ est de rang 2, première classe de Chern nulle et deuxième classe de Chern $c_{2}(c)=n \leq 5$, et $u$ est de degré $d(u) \leq 3$ et caractéristique d'Euler-Poincaré nulle. Nous donnons la série génératrice $P(t)=\sum_{k \geq 0} t^{k} h^{0}\left(\mathrm{M}_{c}, \mathcal{D}_{u}^{\otimes k}\right)$ pour $c_{2}(c)=3, c_{2}(c)=4, d(u)=1$, pour les classes $c$ et $u$ considérées ci-dessus.
\end{abstract}

Texte reçu le 5 mai 2000, accepté le 11 décembre 2000

Gentiana Danila, Institut de Mathématiques de Jussieu, UMR 7586 du CNRS, Case Postale 7012, 2, Place Jussieu, 75251 Paris Cedex 05 (France)

E-mail : gentiana@math.jussieu.fr

Classification mathématique par sujets (2000). — 14D20, 14F05, 14J60.

Mots clefs. - Espaces de modules, fibré déterminant, dualité étrange, séries génératrices. 


\begin{abstract}
Results on the Strange Duality Conjecture on the Projective Plane)
Le Potier's 'Strange Duality' conjecture gives an isomorphism between the space of sections of the determinant bundle on two different moduli spaces of semi-stable sheaves on the complex projective plane $\mathbb{P}_{2}$. We consider two orthogonal classes $c, u$ in the Grothendieck algebra $\mathrm{K}\left(\mathbb{P}_{2}\right)$ such that $c$ is of positive rank and $u$ of rank zero, and we call $\mathrm{M}_{c}$ and $\mathrm{M}_{u}$ the moduli spaces of semi-stable sheaves of class $c$, respectively $u$ on $\mathbb{P}_{2}$. There exists on $M_{c}$ (resp. $M_{u}$ ) a determinant bundle $\mathcal{D}_{u}$ (resp. $\mathcal{D}_{c}$ ) and the product fibre bundle $\mathcal{D}_{c} \otimes \mathcal{D}_{c}$ on the product space $\mathrm{M}_{c} \otimes \mathrm{M}_{c}$ has a canonical section $\sigma_{c, u}$ which provides a linear application $\mathcal{D}_{c, u}: \mathrm{H}^{0}\left(\mathrm{M}_{u}, \mathcal{D}_{c}\right)^{*} \rightarrow \mathrm{H}^{0}\left(\mathrm{M}_{c}, \mathcal{D}_{u}\right)$. If $\mathrm{M}_{c}$ is not empty, $\mathcal{D}_{c, u}$ is conjectured to be an isomorphism. We prove the conjecture in the particular case where $c$ is of rank 2, zero first Chern class and second Chern class $c_{2}(c) \leq 5$, and $u$ is of degree $d(u) \leq 3$ and zero Euler-Poincaré characteristic. In addition we give the generating series $P(t)=\sum_{k>0} t^{k} h^{0}\left(\mathrm{M}_{c}, \mathcal{D}_{u}^{\otimes k}\right)$ for $c_{2}(c)=3$, $c_{2}(c)=4, d(u)=1$, for the particular classes $c$ and $u$ considered above.
\end{abstract}

\title{
1. Introduction
}

La motivation principale de cet article est de fournir des exemples en faveur de la dualité étrange sur le plan projectif conjecturée par Le Potier [14]. On considère l'algèbre de Grothendieck $\mathrm{K}\left(\mathbb{P}_{2}\right)$ des classes de faisceaux algébriques cohérents sur $\mathbb{P}_{2}$, le plan projectif complexe. C'est un groupe abélien isomorphe à $\mathbb{Z}^{3}$, un isomorphisme étant donné par le rang, la classe de Chern et la caractéristique d'Euler-Poincaré (ceci nous permet de désigner chaque classe $c \in \mathrm{K}\left(\mathbb{P}_{2}\right)$ par le triplet formé par son rang $r$, sa première classe de Chern $c_{1}$ et sa caractéristique d'Euler-Poincaré $\chi$, ou bien, lorsque c'est indiqué, sa deuxième classe de Chern $c_{2}$ ). Elle est munie d'une multiplication et d'une forme bilinéaire donnée par $\langle c, u\rangle=\chi(c \cdot u)$. Pour deux classes $c$ et $u$ orthogonales, de rang $r>0$ et respectivement 0 , on note $\mathrm{M}_{c}$ et $\mathrm{M}_{u}$ les espaces de modules des faisceaux semi-stables sur $\mathbb{P}_{2}$ de classe $c$ et respectivement $u$. Sur chacun de ces espaces il existe un fibré inversible $\mathcal{D}_{u}$ et respectivement $\mathcal{D}_{c}$, appelé fibré déterminant. Alors le fibré produit tensoriel externe $\mathcal{D}_{u} \otimes \mathcal{D}_{c}$ sur $\mathrm{M}_{c} \times \mathrm{M}_{u}$ a une section canonique $\sigma_{c, u}$, qui fournit une application linéaire

$$
\mathrm{D}_{c, u}: \mathrm{H}^{0}\left(\mathrm{M}_{u}, \mathcal{D}_{c}\right)^{*} \longrightarrow \mathrm{H}^{0}\left(\mathrm{M}_{c}, \mathcal{D}_{u}\right)
$$

appelée morphisme de dualité étrange. Remarquons que le groupe SL(3) agit sur $\mathbb{P}_{2}$. Il agit ainsi sur les espaces de modules $\mathrm{M}_{c}, \mathrm{M}_{u}$, et sur les fibrés déterminants $\mathcal{D}_{c}, \mathcal{D}_{u}$. Le morphisme $\mathrm{D}_{c, u}$ est un morphisme de $\mathrm{SL}(3)$-représentations.

Conjecture (J. Le Potier). - Si $\mathrm{M}_{c}$ est non-vide, alors le morphisme de dualité étrange est un isomorphisme.

On va se restreindre ici au cas

$$
c=\left(2,0, c_{2}=n\right)
$$

TOME $130-2002-\mathrm{N}^{\mathrm{O}} 1$ 
(qui recouvre le cas où la première classe de Chern est paire), et

$$
u=d(0,1, \chi=0) .
$$

Le cas $d=1$ et $n \leq 19$ a été analysé dans l'article [5]. Le résultat principal est :

ThÉORÈme 1.1. - Soit $\mathfrak{u}=(0,1,0) \in \mathrm{K}\left(\mathbb{P}_{2}\right)$. Si c $=\left(2,0, c_{2}=n\right)$, avec $n \leq 5$ et $u=(0, d, \chi=0)=d \mathfrak{u}$, alors l'application linéaire

$$
D_{c, d \mathfrak{u}}: \mathrm{H}^{0}\left(\mathrm{M}_{d \mathfrak{u}}, \mathcal{D}_{c}\right)^{*} \longrightarrow \mathrm{H}^{0}\left(\mathrm{M}_{c}, \mathcal{D}_{\mathfrak{u}}^{\otimes d}\right)
$$

est un isomorphisme pour $d=2,3$, c'est-à-dire que dans ces conditions la conjecture de dualité étrange est vraie.

On consacre le paragraphe 2 à la construction et l'interprétation géométrique du morphisme $\mathrm{D}_{c, u}$. Les résultats sont contenus dans le théorème 2.1.

Le paragraphe 3 donne des descriptions explicites des faisceaux canoniques sur $\mathrm{M}_{c}$ et $\mathrm{M}_{d \mathfrak{u}}$, en utilisant le théorème de Riemann-Roch-Grothendieck. Ces résultats et le théorème de Kawamata-Viehweg permettent d'obtenir l'annulation de la cohomologie supérieure des fibrés déterminants sur $M_{c}$ et sur $M_{d \mathfrak{u}}$. Ces résultats seront nécessaires aux paragraphes 4 et 7 .

Au paragraphe 4 on utilise [17] pour décrire les espaces de modules $\mathrm{M}_{d \mathfrak{u}}$. Il existe un morphisme $\pi: \mathrm{M}_{d \mathfrak{u}} \rightarrow C_{d}$ (espaces des courbes de degré $d$ dans $\mathbb{P}_{2}$ ) qui associe au faisceau $G$ l'équation de son support schématique. C'est un isomorphisme pour $d=1,2$ et un morphisme dont la fibre générique est de dimension 1 pour $d=3$. Ceci nous permet de calculer $\mathrm{H}^{0}\left(\mathrm{M}_{d \mathfrak{u}}, \mathcal{D}_{c}\right)$.

$\mathrm{Au}$ paragraphe 5 on démontre l'injectivité du morphisme $\mathrm{D}_{c, u}$ en utilisant l'interprétation géométrique du théorème 2.1 (iv). Cela repose sur les propriétés de $\mathrm{M}_{d \mathfrak{u}}$ établies dans le paragraphe 2 .

On calcule au paragraphe 6 les espaces $\mathrm{H}^{0}\left(\mathrm{M}_{c}, \mathcal{D}_{\mathfrak{u}}^{\otimes 2}\right)$ et $\mathrm{H}^{0}\left(\mathrm{M}_{c}, \mathcal{D}_{\mathfrak{u}}^{\otimes 3}\right)$ en tant que SL(3)-représentations, selon la technique développée dans l'article [5] :

Proposition 1.2. - Avec les notations du théorème précédent, le $\mathrm{SL}(3)$ module $\mathrm{H}^{0}\left(\mathrm{M}_{c}, \mathcal{D}_{\mathfrak{u}}^{\otimes 2}\right)$ est isomorphe à $\mathbf{S}^{n}\left(\mathbf{S}^{2} E\right)$ et le $\mathrm{SL}(3)$-module $\mathrm{H}^{0}\left(\mathrm{M}_{c}, \mathcal{D}_{\mathfrak{u}}^{\otimes 3}\right)$ est isomorphe à $\mathbf{S}^{n}\left(\mathbf{S}^{3} E\right) \oplus \mathbf{S}^{n-2}\left(\mathbf{S}^{3} E\right)$ (où $E=\mathrm{H}^{0}\left(\mathbb{P}_{2}, \mathcal{O}(1)\right.$ ) est la représentation standard de $\mathrm{SL}(3))$.

Ceci nous permet de conclure la preuve du théorème. De plus, nous calculons au paragraphe 7 la dimension des espaces de sections de $\mathcal{D}_{\mathfrak{u}}^{\otimes k}$ pour $n=3,4$, qu'on écrit sous forme de série de Poincaré :

ThÉorème 1.3. - (i) Pour l'espace de modules $\mathrm{M}_{(2,0,-1)}$ des faisceaux stables de rang 2 et classes de Chern $(0,3)$, la série de Poincaré de $\mathcal{D}_{\mathfrak{u}}, P(t)=$ $\sum_{k \geq 0} t^{k} h^{0}\left(\mathcal{D}_{\mathfrak{u}}^{\otimes k}\right)$ est donnée par

$$
P(t)=\frac{1+t^{2}+t^{4}}{(1-t)^{10}} .
$$

BULletin DE LA SOCiÉtÉ MATHÉmATiQUe DE FRANCE 
(ii) Pour l'espace de modules $\mathrm{M}_{(2,0,-2)}$ des faisceaux semi-stables de rang 2 et classes de Chern $(0,4)$, la série de Poincaré de $\mathcal{D}_{\mathfrak{u}}$ est donnée par

$$
P(t)=\frac{1+t+7 t^{2}+7 t^{3}+22 t^{4}+7 t^{5}+7 t^{6}+t^{7}+t^{8}}{(1-t)^{14}} .
$$

Notations et conventions. - Le corps de base est le corps des nombres complexes $\mathbb{C}$. Par variété algébrique, on entend schéma de type fini sur $\mathbb{C}$ séparé; les points considérés sont toujours les points fermés. Les résultats restent valables sans changement sur un corps algébriquement clos de caractéristique 0 quelconque. On identifiera la notion de fibré et celle de faisceau localement libre. Pour un espace vectoriel $V$ nous noterons $\mathbb{P}(V)$ l'espace projectif des droites de $V$ et $\mathbb{P}_{\bullet}(V)$ l'espace projectif de Grothendieck des espaces vectoriels quotients de dimension 1.

\section{Morphisme de dualité étrange}

L'objet de cette partie est de présenter la conjecture de Le Potier sur la dualité étrange.

2.1. L'algèbre de Grothendieck $\mathbf{K}\left(\mathbb{P}_{\mathbf{2}}\right)$. — Si $S$ est une variété algébrique, on désigne par $\mathrm{K}(S)$ le groupe de Grothendieck des classes de faisceaux algébriques cohérents sur $S$. Pour un faisceau $F$ on note $[F]$ sa classe dans le groupe $\mathrm{K}(S)$. Dans ce qui suit, on aura à considérer en particulier le groupe de Grothendieck $K\left(\mathbb{P}_{2}\right)$ : c'est un groupe abélien libre de rang 3 ; l'application

$$
\begin{aligned}
\phi: \mathrm{K}\left(\mathbb{P}_{2}\right) & \longrightarrow \mathbb{Z}^{3}, \\
{[F] } & \longmapsto\left(\operatorname{rg}(F), c_{1}(F), \chi(F)\right)
\end{aligned}
$$

qui à la classe d'un faisceau $F$ associe le rang $r$ de $F$, la classe de Chern $c_{1}$ de $F$, et la caractéristique d'Euler-Poincaré $\chi$ de $F$, est un isomorphisme de groupes abéliens. On notera un élément de $K\left(\mathbb{P}_{2}\right)$ par son image $\left(r, c_{1}, \chi\right)$ dans $\mathbb{Z}^{3}$. Si $S$ est lisse, une loi de multiplication sur $\mathrm{K}(S)$ est définie en prolongeant par linéarité la loi de multiplication définie pour $F$ et $G$ faisceaux algébriques cohérents sur $S$ par

$$
F \cdot G=\sum_{p}(-1)^{p} \underline{\operatorname{Tor}}_{p}(F, G)
$$

Ce produit se réduit au produit tensoriel usuel si l'un des deux faisceaux $F$ ou $G$ est localement libre. On parle alors d'algèbre de Grothendieck.

On note $\eta=\left[\mathcal{O}_{\ell}\right]$ la classe du faisceau structural d'une droite $\ell$, et $\eta^{2}=\left[\mathcal{O}_{p}\right]$ celle du faisceau structural d'un point. En tant qu'algèbre, $\mathrm{K}\left(\mathbb{P}_{2}\right)$ est isomorphe à $\mathbb{Z}[\eta] /\left(\eta^{3}\right)$. On munit $\mathrm{K}\left(\mathbb{P}_{2}\right)$ de la forme bilinéaire donnée par $\langle c, u\rangle=\chi(c \cdot u)$. Dans la suite l'orthogonalité sera prise relativement à cette forme. On a aussi sur $K\left(\mathbb{P}_{2}\right)$ une involution $u \mapsto u^{*}$ qui associe à la classe d'un fibré vectoriel celle de son dual.

TOME $130-2002-\mathrm{N}^{\mathrm{O}} 1$ 
2.2. Espaces de modules et fibrés déterminants. - Soit $S$ une variété. On note $\operatorname{pr}_{1}$ la projection $S \times \mathbb{P}_{2} \rightarrow S$ et $\operatorname{pr}_{2}$ la projection $S \times \mathbb{P}_{2} \rightarrow \mathbb{P}_{2}$. Pour un faisceau $\mathcal{F}$ sur $S \times \mathbb{P}_{2}$, on note

$$
\operatorname{pr}_{1 !}([\mathcal{F}]):=\left[R^{0} \operatorname{pr}_{1 *} \mathcal{F}\right]-\left[R^{1} \operatorname{pr}_{1 *} \mathcal{F}\right]+\left[R^{2} \operatorname{pr}_{1 *} \mathcal{F}\right]
$$

dans le groupe $\mathrm{K}(S)$. Cela définit, par linéarité, une application

$$
\mathrm{pr}_{1 !}: \mathrm{K}\left(S \times \mathbb{P}_{2}\right) \longrightarrow \mathrm{K}(S) .
$$

Si $F$ est un faisceau cohérent sur $S$ qui admet une résolution finie par des faisceaux localement libres $A_{i}$ :

$$
0 \rightarrow A_{n} \longrightarrow A_{n-1} \longrightarrow \cdots \longrightarrow A_{0} \rightarrow F \rightarrow 0
$$

on introduit le faisceau inversible

$$
\operatorname{det} F=\operatorname{det} A_{0} \otimes\left(\operatorname{det} A_{1}\right)^{-1} \otimes \cdots \otimes\left(\operatorname{det} A_{n}\right)^{(-1)^{n}} .
$$

L'application qui à $F$ associe son déterminant $\operatorname{det} F$ est multiplicative sur les suites exactes.

Soient $c \in \mathrm{K}\left(\mathbb{P}_{2}\right)$ une classe de Grothendieck de rang $r>0$ et $\mathrm{M}_{c}$ l'espace de modules des faisceaux semi-stables de classe de Grothendieck c. C'est [20] une variété algébrique projective irréductible normale, à singularités rationnelles, de dimension $D=1-\left\langle c^{*}, c\right\rangle$ où $c^{*}$ est la classe duale de $c$. On note $c^{\perp}$ le sousespace de $K\left(\mathbb{P}_{2}\right)$ des classes orthogonales à $c$. Dans [7], Drézet a construit un morphisme surjectif de groupes $\lambda_{c}: c^{\perp} \rightarrow \operatorname{Pic}\left(\mathrm{M}_{c}\right)$ caractérisé par la propriété universelle suivante :

Pour toute famille plate $\mathcal{F}$ de faisceaux semi-stables de classe de Grothendieck $c$, paramétrée par une variété algébrique $S$, la classe

$$
\lambda_{\mathcal{F}}(u)=\operatorname{det} \operatorname{pr}_{1 !}\left(\mathcal{F} \cdot \operatorname{pr}_{2}^{*}(u)\right)
$$

définit un fibré inversible sur la variété $S$. Si $f_{\mathcal{F}}: S \rightarrow \mathrm{M}_{c}$ est le morphisme modulaire associé à la famille $\mathcal{F}$, on a

$$
f_{\mathcal{F}}^{*}\left(\lambda_{c}(u)\right)=\lambda_{\mathcal{F}}(u)
$$

et le fibré $\lambda_{c}(u)$ est le seul à isomorphisme près qui satisfait à cette propriété pour toute famille plate $\mathcal{F}$.

S'il existe un faisceau universel $\mathcal{F}$ sur $\mathrm{M}_{c}$, d'après la propriété universelle de $\lambda_{c}(u)$, il résulte que $\lambda_{c}(u)=\lambda_{\mathcal{F}}(u)$. En général, on prouve l'existence de $\lambda_{c}(u)$ en écrivant $\mathrm{M}_{c}=\Omega^{\mathrm{ss}} / \mathrm{SL}(H)$ comme quotient d'un ouvert dans un schéma de Hilbert par l'action d'un groupe réductif, en considérant une famille universelle sur $\Omega^{\mathrm{ss}}$ et en utilisant un argument de descente.

On note $K_{c}$ le sous- $\mathbb{Z}$-module libre de rang 1 de $c^{\perp}$ des classes de rang 0 . On appelle $\mathfrak{u}$ le générateur positif de $\mathrm{K}_{c}$ (i.e. $c_{1}(\mathfrak{u})$ est un multiple positif de la 
classe hyperplane $h$ dans $\left.\mathrm{H}^{2}\left(\mathbb{P}_{2}, \mathbb{Z}\right)\right)$. On a

$$
\mathfrak{u}=\left(0, \frac{r g(c)}{\delta},-\frac{c_{1}(c)}{\delta}\right)
$$

où $\delta=\operatorname{pgcd}\left(r g(c), c_{1}(c)\right)$.

Le fibré $\mathcal{D}=\mathcal{D}_{\mathfrak{u}}=\lambda_{c}(-\mathfrak{u})$ s'appelle fibré déterminant de Donaldson sur $\mathrm{M}_{c}$. Pour toute classe $u \in \mathrm{K}_{c}$, on introduit plus généralement le fibré inversible $\mathcal{D}_{u}=\lambda_{c}(-u)$; c'est donc un multiple du fibré déterminant de Donaldson.

Le problème du calcul de la dimension de l'espace de sections $\mathrm{H}^{0}\left(\mathrm{M}_{c}, \mathcal{D}_{u}\right)$ a conduit Le Potier [17] à introduire $\mathrm{M}_{u}$, l'espace de modules des faisceaux semi-stables de dimension 1 de classe de Grothendieck $u$. La notion de semistabilité (resp. stabilité) se généralise ( $c f .[17])$ pour les faisceaux algébriques cohérents $F$ de dimension 1 . L'espace $\mathrm{M}_{u}$ est encore une variété algébrique projective irréductible normale à singularités rationnelles. La classe $c$ (appartenant à $u^{\perp}$ ) permet de construire un fibré inversible $\mathcal{D}_{c}=\lambda_{u}(-c)$ sur $\mathrm{M}_{u}$, de la même manière que sur $\mathrm{M}_{c}$. Dans certains cas l'espace $\mathrm{M}_{u}$ et le fibré $\mathcal{D}_{c}$ sont plus faciles à décrire.

2.3. Construction du morphisme de dualité étrange. - On considère deux classes $c, u \in \mathrm{K}\left(\mathbb{P}_{2}\right)$ dans l'algèbre de Grothendieck . On suppose qu'elles sont orthogonales, que $r(c)>0$, que $r(u)=0$ et $c_{1}(u)>0$. Le morphisme de dualité étrange est une conséquence de la construction simultanée des fibrés déterminants $\mathcal{D}_{u}$ sur $\mathrm{M}_{c}$ et $\mathcal{D}_{c}$ sur $\mathrm{M}_{u}$. Sa construction et son interprétation géométrique sont résumées dans le théorème suivant :

THÉORÈme 2.1. - (i) Il existe une section canonique, définie à une constante près, $\sigma_{c, u} \in \mathrm{H}^{0}\left(\mathrm{M}_{c} \times \mathrm{M}_{u}, \mathcal{D}_{u} \otimes \mathcal{D}_{c}\right)$ qui s'annule exactement aux points $([F],[G])$ tels que $h^{0}\left(\mathbb{P}_{2}, F \otimes G\right)=h^{1}\left(\mathbb{P}_{2}, F \otimes G\right) \neq 0$.

(ii) La section $\sigma_{c, u}$ définit une application linéaire

$$
\mathrm{D}_{c, u}: \mathrm{H}^{0}\left(\mathrm{M}_{u}, \mathcal{D}_{c}\right)^{*} \longrightarrow \mathrm{H}^{0}\left(\mathrm{M}_{c}, \mathcal{D}_{u}\right) .
$$

(iii) On note $\sigma_{F}$ la restriction de $\sigma_{c, u} \grave{a}\{[F]\} \times \mathrm{M}_{u}$. Si $\sigma_{c, u}$ n'est pas identiquement nulle, l'association $F \mapsto\left[\sigma_{F}\right]$ définit une application rationnelle

$$
\Phi: \mathrm{M}_{c} \rightarrow \mathbb{P H}^{0}\left(\mathrm{M}_{u}, \mathcal{D}_{c}\right) .
$$

Si en outre $\sigma_{F}$ n'est pas identiquement nulle pour tout $[F] \in \mathrm{M}_{c}$, l'application $\Phi$ est régulière.

(iv) Si l'image du morphisme $\Phi$ n'est pas contenue dans un hyperplan l'application $\mathrm{D}_{c, u}$ est injective.

La conjecture de Le Potier est alors :

Conjecture 2.2. - Si $\mathrm{M}_{c}$ est non-vide alors le morphisme $\mathrm{D}_{c, u}$ de dualité étrange est un isomorphisme.

TOME $130-2002-\mathrm{N}^{\mathrm{O}} 1$ 
Preuve du théorème 2.1. - On commence par rappeler les résultats suivants :

Lemme 2.3. - Soit $S$ une variété algébrique. Soient $\mathcal{F}$ et $\mathcal{G}$ des familles plates de faisceaux semi-stables sur $\mathbb{P}_{2}$ de classes de Grothendieck c et u respectivement, paramétrées par $S$. Alors :

a) Le faisceau $\mathcal{G}$ a une résolution

$$
0 \rightarrow \mathcal{Q} \longrightarrow \mathcal{R} \longrightarrow \mathcal{G} \rightarrow 0
$$

sur $S \times \mathbb{P}_{2}$ par des faisceaux localement libres $\mathcal{Q}$ et $\mathcal{R}$.

b) Le faisceau $\mathcal{F}$ a une résolution

$$
0 \rightarrow \mathcal{A} \longrightarrow \mathcal{B} \longrightarrow \mathcal{F} \rightarrow 0
$$

sur $S \times \mathbb{P}_{2}$ par des faisceaux localement libres $\mathcal{A}$ et $\mathcal{B}$. En plus, on peut choisir $\mathcal{B}$ tel que

$$
h^{0}\left(\mathcal{B}_{s} \otimes \mathcal{G}_{s}\right)=0 \quad \text { pour tout } s \in S .
$$

c) $\underline{\operatorname{Tor}}_{i}(\mathcal{F}, \mathcal{G})=0$ pour $i>0$.

Preuve. - La résolution pour $\mathcal{G}$ résulte du fait que $\mathcal{G}$ est pur de dimension 1 sur chaque fibre. La résolution pour $\mathcal{F}$ résulte du fait que la restriction de $\mathcal{F}$ à chaque fibre est un faisceau sans torsion sur $\mathbb{P}_{2}$. On peut changer le faisceau $\mathcal{B}$ dans la résolution de $\mathcal{F}$ en $\left[\mathcal{B} \otimes p r_{2}^{*} \mathcal{O}(-n)\right]^{m}$, pour $n \geq 0$ et $m$ assez grand. Pour un choix de $n$ assez grand on obtient $h^{0}\left(\mathcal{B}_{s} \otimes \mathcal{G}_{s}\right)=0$ pour tout $s$. Il résulte d'après a), b), que $\underline{\operatorname{Tor}}_{i}(\mathcal{F}, \mathcal{G})=0$ pour $i \geq 2$ et que $\underline{\operatorname{Tor}}_{1}(\mathcal{F}, \mathcal{G})$ est inclus dans $\mathcal{G} \otimes \mathcal{A}$ et dans $\mathcal{F} \otimes \mathcal{Q}$. Comme $\mathcal{G} \otimes \mathcal{A}$ est de torsion, et $\mathcal{F} \otimes \mathcal{Q}$ sans torsion, on en déduit que $\underline{\operatorname{Tor}}_{1}(\mathcal{F}, \mathcal{G})=0$.

Soient $S, \mathcal{F}, \mathcal{G}$ comme dans le lemme. D'après c), on a une suite exacte courte

$$
0 \rightarrow \mathcal{A} \otimes \mathcal{G} \stackrel{a}{\longrightarrow} \mathcal{B} \otimes \mathcal{G} \longrightarrow \mathcal{F} \otimes \mathcal{G} \rightarrow 0 .
$$

On considère son image directe par $\operatorname{pr}_{1 *}$. Le lemme $2.3 \mathrm{~b}$ ) conduit à

$$
\operatorname{pr}_{1 *}(\mathcal{A} \otimes \mathcal{G})=\operatorname{pr}_{1 *}(\mathcal{B} \otimes \mathcal{G})=0 .
$$

Le faisceau $\mathcal{G}$ est de dimension 1 dans les fibres, donc

$$
R^{2} \operatorname{pr}_{1 *}(\mathcal{A} \otimes \mathcal{G})=R^{2} \operatorname{pr}_{1 *}(\mathcal{B} \otimes \mathcal{G})=0 .
$$

Alors la suite

$$
\begin{aligned}
0 \rightarrow \operatorname{pr}_{1 *}(\mathcal{F} \otimes \mathcal{G}) \longrightarrow R^{1} \operatorname{pr}_{1 *}(\mathcal{A} \otimes \mathcal{G}) & \stackrel{a}{\longrightarrow} R^{1} \operatorname{pr}_{1 *}(\mathcal{B} \otimes \mathcal{G}) \\
& \longrightarrow R^{1} \operatorname{pr}_{1 *}(\mathcal{F} \otimes \mathcal{G}) \rightarrow 0
\end{aligned}
$$

est exacte.

LEMME 2.4. - Les faisceaux $R^{1} \operatorname{pr}_{1 *}(\mathcal{A} \otimes \mathcal{G})$ et $R^{1} \operatorname{pr}_{1 *}(\mathcal{B} \otimes \mathcal{G})$ sont localement libres de même rang sur $S$. 
Preuve. - Puisque les familles $\mathcal{F}$ et $\mathcal{G}$ sont $S$-plates, on obtient que $\mathcal{A} \otimes \mathcal{G}, \mathcal{B} \otimes \mathcal{G}$ et $\mathcal{F} \otimes \mathcal{G}$ sont des familles $S$-plates. Alors les suites (1), (4), (5) sont compatibles avec les changements de base $S^{\prime} \rightarrow S$. En particulier, pour $S^{\prime}=\{s\} \in S$, on obtient à partir de la suite (5) une suite exacte

(6) $0 \rightarrow \mathrm{H}^{0}\left(\mathcal{F}_{s} \otimes \mathcal{G}_{s}\right) \longrightarrow \mathrm{H}^{1}\left(\mathcal{A}_{s} \otimes \mathcal{G}_{s}\right) \stackrel{a_{s}}{\longrightarrow} \mathrm{H}^{1}\left(\mathcal{B}_{s} \otimes \mathcal{G}_{s}\right) \longrightarrow \mathrm{H}^{1}\left(\mathcal{F}_{s} \otimes \mathcal{G}_{s}\right) \rightarrow 0$

et

$$
h^{0}\left(\mathcal{A}_{s} \otimes \mathcal{G}_{s}\right)=h^{0}\left(\mathcal{B}_{s} \otimes \mathcal{G}_{s}\right)=h^{2}\left(\mathcal{A}_{s} \otimes \mathcal{G}_{s}\right)=h^{2}\left(\mathcal{B}_{s} \otimes \mathcal{G}_{s}\right)=0
$$

Ces annulations et le fait que les familles $\mathcal{A} \otimes \mathcal{G}$ et $\mathcal{B} \otimes \mathcal{G}$ sont plates sur $S$ nous assurent que $R^{1} \operatorname{pr}_{1 *}(\mathcal{A} \otimes \mathcal{G})$ est localement libre de rang

$$
h^{1}\left(\mathcal{A}_{s} \otimes \mathcal{G}_{s}\right)=-\chi\left(\mathcal{A}_{s} \otimes \mathcal{G}_{s}\right)
$$

Le même argument montre que $R^{1} \operatorname{pr}_{1 *}(\mathcal{B} \otimes \mathcal{G})$ est localement libre de rang

$$
-\chi\left(\mathcal{B}_{s} \otimes \mathcal{G}_{s}\right) \text {. }
$$

La suite (6) implique

$$
\chi\left(\mathcal{A}_{s} \otimes \mathcal{G}_{s}\right)-\chi\left(\mathcal{B}_{s} \otimes \mathcal{G}_{s}\right)=\chi\left(\mathcal{F}_{s} \otimes \mathcal{G}_{s}\right) .
$$

Le lemme $2.3 \mathrm{c})$ implique $\left[\mathcal{F}_{s} \otimes \mathcal{G}_{s}\right]=\left[\mathcal{F}_{s}\right] \cdot\left[\mathcal{G}_{s}\right]$ dans $\mathrm{K}\left(\mathbb{P}_{2}\right)$. Comme $c$ et $u$ sont orthogonales, on a $\chi\left(\mathcal{F}_{s} \otimes \mathcal{G}_{s}\right)=0$. Alors les faisceaux $R^{1} \operatorname{pr}_{1 *}(\mathcal{A} \otimes \mathcal{G})$ et $R^{1} \operatorname{pr}_{1 *}(\mathcal{B} \otimes \mathcal{G})$ ont même rang.

En utilisant la suite exacte (5), on définit un fibré $\mathcal{D}_{S}$ par

$$
\mathcal{D}_{S}:=\left[\operatorname{det} \operatorname{pr}_{1 !}(\mathcal{F} \cdot \mathcal{G})\right]^{(-1)}=\operatorname{det} R^{1} \operatorname{pr}_{1 *}(\mathcal{B} \otimes \mathcal{G}) \otimes\left[\operatorname{det} R^{1} \operatorname{pr}_{1 *}(\mathcal{A} \otimes \mathcal{G})\right]^{(-1)}
$$

L'application $a$ fournit une section $\sigma_{S}$ de ce fibré inversible sur $S$. Ni le fibré $\mathcal{D}_{S}$, ni la section $\sigma_{S}$, à une fonction inversible près, ne dépendent de la résolution choisie. La suite exacte (6) montre que la section $\sigma_{S}$ s'annule exactement aux points $s \in S$ où l'application linéaire $a_{\left.\right|_{s}}$ n'est pas inversible. Ces points sont ceux où $h^{0}\left(\mathcal{F}_{s} \otimes \mathcal{G}_{s}\right)=h^{1}\left(\mathcal{F}_{s} \otimes \mathcal{G}_{s}\right) \neq 0$.

On a vu que la suite (5) était compatible avec les changements de base $S^{\prime} \rightarrow S$. Il résulte que le fibré inversible $\mathcal{D}_{S}$ et la section $\sigma_{S}$ le sont aussi. Soit $\phi: S \rightarrow \mathrm{M}_{c} \times \mathrm{M}_{u}$ le morphisme modulaire associé aux familles $\mathcal{F}, \mathcal{G}$.

Proposition 2.5. - Il existe un fibré inversible $\mathcal{D}_{c, u}$ sur $\mathrm{M}_{c} \times \mathrm{M}_{u}$ et une section $\sigma_{c, u}$ bien déterminée à une constante multiplicative près, qui vérifie : pour toute variété algébrique $S$ et pour toutes familles plates $\mathcal{F}, \mathcal{G}$ de faisceaux semi-stables de classes c respectivement $u$ dans $\mathrm{K}\left(\mathbb{P}_{2}\right)$, paramétrées par $S$, on a

$$
\mathcal{D}_{S}=\phi^{*}\left(\mathcal{D}_{c, u}\right) \quad \text { et } \quad \sigma_{S}=\phi^{*}\left(\sigma_{c, u}\right) \quad \grave{a} \text { une fonction inversible près. }
$$

TOME $130-2002-\mathrm{N}^{\mathrm{O}} 1$ 
Preuve. - La preuve est classique. On commence par les deux lemmes suivants.

LEMme 2.6 (cf. [26], [15], [20]). — Il existe une variété lisse $\Omega_{c}$, une famille plate $\mathcal{F}_{c}$ de faisceaux semi-stables de classe c sur $\mathbb{P}_{2}$ paramétrée par $\Omega_{c}$ et un groupe réductif $G_{c}$ qui agit sur $\Omega_{c}$ tels que $\mathrm{M}_{c}$ soit un bon quotient de $\Omega_{c}$ sous l'action de $G_{c}$.

La description de $\Omega_{c}$ est la suivante. On introduit pour $m$ entier assez grand la caractéristique d'Euler-Poincaré $P(m)$ de $c(m)$, et la somme directe $B$ de $N=P(m)$ exemplaires du fibré inversible $\mathcal{O}_{\mathbb{P}_{2}}(-m)$. On considère le schéma de Hilbert $\operatorname{Hilb}^{c}(B)$ des faisceaux cohérents $F$ de classe de Grothendieck $c$ quotients de $B$. Le groupe $G_{c}:=\operatorname{Aut}(B)$ opère de manière naturelle sur $\operatorname{Hilb}^{c}(B)$. On note $\Omega_{c}:=\Omega^{\text {ss }}$ l'ouvert des points semi-stables pour l'action de $G_{c}$. Ces points représentent les faisceaux semi-stables $F$ pour lesquels le morphisme naturel $\mathrm{H}^{0}(B(m)) \rightarrow \mathrm{H}^{0}(F(m))$ est un isomorphisme. L'ouvert $\Omega^{\mathrm{ss}}$ est invariant par l'action du groupe réductif $\operatorname{Aut}(B)$. C'est un ouvert lisse et sur $\Omega^{\text {ss }} \times \mathbb{P}_{2}$ on dispose d'un faisceau quotient universel $\mathcal{F}$, lequel est aussi muni d'une action de $G_{c}$. L'ouvert $\Omega^{\mathrm{ss}}$ admet pour bon quotient l'espace de modules grossier $\mathrm{M}_{c}$ des faisceaux semi-stables de classe $c$.

LEMME 2.7 (cf. [17]). — Il existe une variété lisse $\Omega_{u}$, une famille plate $\mathcal{G}_{u}$ de faisceaux semi-stables de classe $u$ sur $\mathbb{P}_{2}$ paramétrée par $\Omega_{u}$ et un groupe réductif $G_{u}$ qui agit sur $\Omega_{u}$, tels que $\mathrm{M}_{u}$ soit un bon quotient de $\Omega_{u}$ sous l'action de $G_{u}$.

La description de la variété $\mathrm{M}_{u}$ est analogue à celle de la variété $\mathrm{M}_{c}$. Il existe un entier $m$ suffisamment grand pour que tout faisceau semi-stable $G$ sur $\mathbb{P}_{2}$ de classe $u=(0, d, \chi) \in \mathrm{K}\left(\mathbb{P}_{2}\right)$ vérifie : le faisceau $G(m)$ est engendré par ses sections globales et $\mathrm{H}^{1}(G(m))=0$. On considère $H$ un espace vectoriel de dimension $n=d m+\chi$ sur $\mathbb{C}$. Soit $\operatorname{Hilb}^{u}(B)$ le schéma de HilbertGrothendieck des faisceaux quotients de $B=H \otimes \mathcal{O}(-m)$ de classe $u \in \mathrm{K}\left(\mathbb{P}_{2}\right)$. Le groupe $G_{u}:=\operatorname{GL}(H)$ opère de manière naturelle sur $\operatorname{Hilb}^{u}(B)$. On considère l'ouvert $\Omega_{u}:=\Omega^{\mathrm{ss}}$ des points semi-stables pour l'action de $G_{u}$ : ces points correspondent aux faisceaux quotients de $B$ qui sont semi-stables et tels que le morphisme d'évaluation $H \rightarrow \mathrm{H}^{0}(F(m))$ soit un isomorphisme. Cet ouvert est lisse et $\mathrm{M}_{u}$ est le bon quotient de $\Omega^{\mathrm{ss}}$ pour l'action du groupe $G_{u}$.

On note $\rho$ la projection $\Omega_{c} \times \Omega_{u} \rightarrow \mathrm{M}_{c} \times \mathrm{M}_{u}$. On considère la construction précédente de $\mathcal{D}_{S}$ dans le cas où $S:=\Omega_{c} \times \Omega_{u}, \mathcal{F}:=\operatorname{pr}_{13}^{*}\left(\mathcal{F}_{c}\right), \mathcal{G}:=\operatorname{pr}_{23}^{*}\left(\mathcal{G}_{u}\right)$, où $\operatorname{pr}_{13}: \Omega_{c} \times \Omega_{u} \times \mathbb{P}_{2} \rightarrow \Omega_{c} \times \mathbb{P}_{2}$ et $\mathrm{pr}_{23}: \Omega_{c} \times \Omega_{u} \times \mathbb{P}_{2} \rightarrow \Omega_{u} \times \mathbb{P}_{2}$ sont les projections. On note $\mathcal{D}_{\Omega}$ le fibré déterminant sur $\Omega_{c} \times \Omega_{u}$ ainsi obtenu et $\sigma_{\Omega}$ sa section canonique, à une fonction inversible près.

De la compatibilité du fibré $\mathcal{D}_{S}$ aux changements de base $S^{\prime} \rightarrow S$, il résulte une action du groupe $G_{c} \times G_{u}$ sur le fibré $\mathcal{D}_{\Omega}$. La section $\sigma_{\Omega}$ est équivariante. 
Dans le lemme qui suit on vérifie que la condition de descente est satisfaite pour $\mathcal{D}_{\Omega}$.

LEMME 2.8. - Soit $\left(s_{c}, s_{u}\right)$ un point de $\Omega_{c} \times \Omega_{u}$ tel que l'orbite $G_{c} \cdot s_{c} \times G_{u} \cdot s_{u}$ soit fermée dans $\Omega_{c} \times \Omega_{u}$. Alors le stabilisateur $G_{s_{c}} \times G_{s_{u}}$ du point $\left(s_{c}, s_{u}\right)$ agit trivialement sur la fibre $\mathcal{D}_{\left(s_{c}, s_{u}\right)}$ du fibré $\mathcal{D}_{\Omega}$ au point $\left(s_{c}, s_{u}\right)$.

Preuve. - Les points $\left(s_{c}, s_{u}\right)$ d'orbite fermée sont les points pour lesquels l'orbite $G_{c} \cdot s_{c}$ est fermée dans $\Omega_{c}$ et l'orbite $G_{u} \cdot s_{u}$ est fermée dans $\Omega_{u}$. D'après le lemme 4.2 de [8], le faisceau $\mathcal{F}_{s_{c}}$ sur $\mathbb{P}_{2}$ est somme directe de faisceaux stables de même polynôme de Hilbert réduit, et de même pour $\mathcal{G}_{s_{u}}$. Écrivons :

$$
\mathcal{F}_{s_{c}}=F_{1}^{m_{1}} \oplus \cdots \oplus F_{k}^{m_{k}}, \quad \mathcal{G}_{s_{u}}=G_{1}^{n_{1}} \oplus \cdots \oplus G_{\ell}^{n_{\ell}}
$$

pour des faisceaux stables $F_{i}, G_{j}$ différents deux par deux. Le stabilisateur du point $\left(s_{c}, s_{u}\right)$ est

$$
G_{s_{c}} \times G_{s_{u}}=\mathrm{GL}\left(m_{1}\right) \times \cdots \times \mathrm{GL}\left(m_{k}\right) \times \mathrm{GL}\left(n_{1}\right) \times \cdots \times \mathrm{GL}\left(n_{\ell}\right) .
$$

D'après la définition de $\mathcal{D}_{S}$ on a

$$
\mathcal{D}_{\left(s_{c}, s_{u}\right)}=\left[\operatorname{det} H^{0}\left(\mathbb{P}_{2}, \mathcal{F}_{s_{c}} \otimes \mathcal{G}_{s_{u}}\right)\right]^{-1} \otimes \operatorname{det} H^{1}\left(\mathbb{P}_{2}, \mathcal{F}_{s_{c}} \otimes \mathcal{G}_{s_{u}}\right) .
$$

On a également

$$
\mathrm{H}^{q}\left(\mathbb{P}_{2}, \mathcal{F}_{s_{c}} \otimes \mathcal{G}_{s_{u}}\right)=\bigoplus_{i=1}^{k} \bigoplus_{j=1}^{\ell} \mathrm{H}^{q}\left(\mathbb{P}_{2}, F_{i}^{m_{i}} \otimes G_{j}^{n_{j}}\right)
$$

pour $q=0,1$. Par conséquent, l'élément $\left(g_{1}, \ldots, g_{k}, h_{1}, \ldots, h_{\ell}\right)$ appartenant à $G_{s_{c}} \times G_{s_{u}}$, agit sur $\mathcal{D}_{\left(s_{c}, s_{u}\right)}$ par multiplication avec

$$
\prod_{i=1}^{k} \prod_{j=1}^{\ell}\left(\operatorname{det} g_{i} \cdot \operatorname{det} h_{j}\right)^{-h^{0}\left(\mathbb{P}_{2}, F_{i} \otimes G_{j}\right)+h^{1}\left(\mathbb{P}_{2}, F_{i} \otimes G_{j}\right)} \text {. }
$$

Les faisceaux $F_{i}, G_{j}$ ont le même polynôme de Hilbert réduit que $\mathcal{F}_{s_{c}}$ respectivement $\mathcal{G}_{s_{u}}$. Puisque les classes $c$ et $u$ sont orthogonales, on obtient

$$
h^{0}\left(\mathbb{P}_{2}, F_{i} \otimes G_{j}\right)=h^{1}\left(\mathbb{P}_{2}, F_{i} \otimes G_{j}\right) .
$$

Donc le stabilisateur du point $\left(s_{c}, s_{u}\right)$ agit trivialement sur $\mathcal{D}_{\left(s_{c}, s_{u}\right)}$.

Le lemme de Kempf (lemme de descente, th. 2.3 de [8]) implique l'existence d'un unique fibré inversible $\mathcal{D}_{c, u}$ sur $\mathrm{M}_{c} \times \mathrm{M}_{u}$ qui satisfait $\rho^{*}\left(\mathcal{D}_{c, u}\right)=\mathcal{D}_{\Omega}$. Mais $\mathrm{M}_{c} \times \mathrm{M}_{u}$ est $u$ bon quotient de $\Omega_{c} \times \Omega_{u}$ par l'action du groupe $G_{c} \times G_{u}$, donc

$$
\mathrm{H}^{0}\left(\mathrm{M}_{c} \times \mathrm{M}_{u}, \mathcal{D}_{c, u}\right)=\mathrm{H}^{0}\left(\Omega_{c} \times \Omega_{u}, \mathcal{D}_{\Omega}\right)^{G_{c} \times G_{u}} .
$$

On peut choisir une résolution (2) $G_{c}$-équivariante. La suite exacte (5) sera alors $G_{c} \times G_{u}$-équivariante. Il résulte que la section $\sigma_{\Omega}$, bien déterminée à fonction inversible près, est $G_{c} \times G_{u}$-équivariante, et donc bien déterminée à

TOME $130-2002-\mathrm{N}^{\mathrm{O}} 1$ 
une constante multiplicative près. On déduit l'existence d'une section $\sigma_{c, u} \in$ $\mathrm{H}^{0}\left(\mathrm{M}_{c} \times \mathrm{M}_{u}, \mathcal{D}_{c, u}\right)$, bien déterminée à une constante multiplicative près.

Le fait que le fibré $\mathcal{D}_{c, u}$ et la section $\sigma_{c, u}$ satisfont la propriété d'universalité de l'énoncé résulte d'un argument classique ( $c f$. [16], §2.13). Cela termine la preuve de la proposition 2.5 .

LEMME 2.9. - Le fibré inversible $\mathcal{D}_{c, u}$ sur $\mathrm{M}_{c} \times \mathrm{M}_{u}$ est isomorphe au fibré inversible $\mathcal{D}_{u} \otimes \mathcal{D}_{c}$.

Preuve. - Prouvons que la restriction $\mathcal{D}_{c, s_{u}}$ du fibré $\mathcal{D}_{c, u}$ à $\mathrm{M}_{c} \times\left\{s_{u}\right\}$ est isomorphe au fibré $\mathcal{D}_{u}$ pour tout $s \in \mathrm{M}_{u}$. Soit $G$ un faisceau sur $\mathbb{P}_{2}$ dans la classe du point $s_{u}$. Soit $S$ une variété et $\mathcal{F}$ une famille plate de faisceaux semi-stables de classe $c \in \mathrm{K}\left(\mathbb{P}_{2}\right)$, paramétrée par $S$. On note $\varphi: S \rightarrow \mathrm{M}_{c}$ le morphisme modulaire associé à $\mathcal{F}$ et $\phi=\varphi \times s_{u}: S \rightarrow \mathrm{M}_{c} \times \mathrm{M}_{u}$ le morphisme modulaire associé à $\mathcal{F}, G$. D'après la propriété d'universalité on a

$$
\varphi^{*} \mathcal{D}_{c, s_{u}}=\phi^{*} \mathcal{D}_{c, u}=\operatorname{det} \operatorname{pr}_{1 !}\left(\mathcal{F} \otimes \operatorname{pr}_{2}^{*}(G)\right)^{-1} \text {. }
$$

Alors il y a un isomorphisme $\varphi^{*} \mathcal{D}_{c, s_{u}} \simeq \varphi^{*} \mathcal{D}_{c, u}$ pour tout couple $(S, \mathcal{F})$. On déduit que $\mathcal{D}_{c, s_{u}}$ et $\mathcal{D}_{u}$ sont isomorphes. Nous prouvons de la même manière que la restriction de $\mathcal{D}_{c, u}$ à $\left\{s_{u}\right\} \times \mathrm{M}_{u}$ est isomorphe au fibré $\mathcal{D}_{c}$ sur $\mathrm{M}_{c}$. Les variétés $\mathrm{M}_{u}$ et $\mathrm{M}_{c}$ sont projectives et intègres. Le lemme suivant s'applique.

LEMME 2.10 (voir [24], p. 23, [23], §5, th. 5.1 et cor. 5.2)

Soit $M$ une variété algébrique intègre, $N$ une variété algébrique projective, et $\mathcal{L}$ un faisceau inversible sur $M \times N$. On suppose que la classe d'isomorphisme de la restriction de $\mathcal{L}$ à la fibre $\{m\} \times N$ est la même pour chaque $m \in M$. Alors $\mathcal{L}$ s'écrit $L_{1} \otimes L_{2}$ pour deux faisceaux $L_{1} \in \operatorname{Pic}(M), L_{2} \in \operatorname{Pic}(N)$.

Du lemme et du calcul des restrictions du fibré $\mathcal{D}_{c, u}$ aux fibres on trouve $\mathcal{D}_{c, u} \simeq \mathcal{D}_{u} \otimes \mathcal{D}_{c}$.

Cela prouve le point (i) du théorème 2.1. Les points (ii) et (iii) sont évidents. Le point (iv) résulte du lemme géométrique évident :

LEMme 2.11. - Soient $M$ et $N$ deux variétés projectives, $\mathcal{D}$ et $\mathcal{E}$ des fibrés inversibles sur $M$ respectivement $N$, et une section $\sigma \in \mathrm{H}^{0}(M \times N, \mathcal{D} \otimes \mathcal{E})$. On note $\sigma_{m} \in \Gamma(N, \mathcal{E})$ la restriction de $\sigma \grave{a}\{m\} \times N$. On suppose que $\sigma_{m}$ n'est pas identiquement nulle pour tout $m \in M$. Alors :

(i) La section $\sigma$ produit un morphisme $D_{M, N}: \mathrm{H}^{0}(N, \mathcal{E})^{*} \rightarrow \mathrm{H}^{0}(M, \mathcal{D})$.

(ii) La section $\sigma$ produit une application $\Phi: M \rightarrow \mathbb{P H}^{0}(N, \mathcal{E})$ définie par $m \mapsto\left[\sigma_{m}\right]$ qui vérifie $\Phi^{*} \mathcal{O}(1)=\mathcal{D}$.

(iii) L'application $\Phi$ induit sur les sections globales une application $\Phi^{*}$ : $\mathrm{H}^{0}(N, \mathcal{E})^{*} \rightarrow \mathrm{H}^{0}(M, \mathcal{D})$. Alors $\Phi^{*}=D_{M, N}$.

(iv) Si l'image du morphisme $\Phi$ n'est pas contenue dans un hyperplan (c'està-dire que les $\sigma_{m}$ engendrent $\mathrm{H}^{0}(N, \mathcal{E})$ ), alors $D_{M, N}$ est injectif.

BULLETIN DE LA SOCIÉtÉ MATHÉMATIQUE DE FRANCE 
On applique le lemme pour $M=\mathrm{M}_{c}, N=\mathrm{M}_{u}, \mathcal{D}=\mathcal{D}_{u}, \mathcal{E}=\mathcal{D}_{c}, \sigma=\sigma_{c, u}$, lorsque $\sigma_{F}$ n'est pas identiquement nulle pour tout $[F] \in \mathrm{M}_{c}$.

Remarque 2.12. - 1) D'après le point (i) du théorème 2.1, le fait que $\sigma_{F}$ n'est pas identiquement nulle équivaut à : il existe $G \in \mathrm{M}_{u}$ tel que $h^{0}(F \otimes G)=$ $h^{1}(F \otimes G)=0$. Dans les situations considérées cela sera vrai pour tout $F \in \mathrm{M}_{c}$. Le Potier a montré cette affirmation si $2 c_{1}^{c}=0 \bmod r^{c}$, en utilisant l'existence d'une droite, ou bien d'une conique qui n'est pas de saut pour un faisceau stable générique $F$ de classe $c$.

2) Dans la proposition 3.3 de [19], pour une classe $c$ donnée telle que l'espace $M_{c}$ est non-vide, on montre l'existence de $u \in c^{\perp}$ de dimension 1 telle que $\sigma_{c, u} \neq 0$. La démonstration repose sur un théorème de Flenner, qui donne le comportement de la semi-stabilité par restriction aux courbes de degré élevé, et la version effective d'un résultat de Faltings.

\section{Préliminaires : le faisceau canonique sur $M_{d \mathfrak{u}}$ et sur $M_{c}$}

Le but de ce paragraphe est de donner des descriptions explicites pour les faisceaux canoniques sur $\mathrm{M}_{d \mathfrak{u}}$ et sur $\mathrm{M}_{c}$. Ces résultats, et le théorème d'annulation de Kawamata-Viehweg, seront appliqués pour déduire des résultats d'annulation (th. 3.8, prop. 4.9).

Nous allons étudier $\mathrm{M}_{u}=\mathrm{M}_{d \mathfrak{u}}$, pour $u=d \mathfrak{u}=d(0,1,0)$ et $d \geq 1$. On définit $C_{d}=\mathbb{P H}^{0}\left(\mathbb{P}_{2}, \mathcal{O}(d)\right)$, l'espace des courbes de degré $d$ dans $\mathbb{P}_{2}$. Pour un faisceau $G \in \mathrm{M}_{u}$, il existe une présentation

$$
0 \rightarrow Q \stackrel{a}{\longrightarrow} R \longrightarrow G \rightarrow 0
$$

et det $a \in \mathrm{H}^{0}\left(\mathbb{P}_{2}\right.$, det $\left.R \otimes \operatorname{det} Q^{-1}\right)=\mathrm{H}^{0}\left(\mathbb{P}_{2}, \mathcal{O}(d)\right)$ s'appelle l'équation du support schématique de $G$. Cela ne dépend pas, à une constante près, du choix de $Q, R$. On définit ainsi une application

$$
\pi: \mathrm{M}_{u} \longrightarrow C_{d} .
$$

3.1. Le faisceau canonique sur $\mathbf{M}_{d \mathfrak{u}}$. - La description de la variété $\mathrm{M}_{d \mathfrak{u}}$ comme quotient d'un ouvert lisse $\Omega^{\text {ss }}$ d'un schéma de Hilbert-Grothendieck par l'action d'un groupe réductif $\mathrm{GL}(H)$ a été donnée au $§ 2.3$, lemme 2.7.

Proposition 3.1. - Le faisceau dualisant de la variété $\mathrm{M}_{u}, \omega=\omega_{\mathrm{M}_{u}}$, est inversible et isomorphe à $\pi^{*} \mathcal{O}(-3 d)$.

Preuve. - On peut supposer $d \geq 3$, puisque pour $d=1,2, \pi$ est un isomorphisme (voir prop. 4.4) et l'égalité est vérifiée. Il résulte du théorème de Boutot [4], que $\mathrm{M}_{u}$ est une variété à singularités rationnelles. En particulier c'est une variété normale et de Cohen-Macaulay. Soient $\mathrm{M}_{u}^{s}$ l'ouvert de $\mathrm{M}_{u}$ des classes de faisceaux stables, $j$ l'inclusion canonique $j: \mathrm{M}_{u}^{s} \rightarrow \mathrm{M}_{u}$ et $Y=C_{\mathrm{M}_{u}^{s}}$ le complémentaire de $\mathrm{M}_{u}^{s}$ dans $\mathrm{M}_{u}$. Il est démontré dans [17], prop 3.4, que

TOME $130-2002-\mathrm{N}^{\mathrm{O}} 1$ 
codim $Y \geq 2$. Soit $\underline{p} \in Y$ le point générique d'une sous-variété irréductible de $Y$. Puisque $\mathrm{M}_{u}$ est de Cohen-Macaulay on obtient $\operatorname{prof}\left(\omega_{\underline{p}}\right) \geq 2$. Or on a l'énoncé suivant (voir [11]) :

THÉORÈme 3.2. - Soit $X$ un schéma et $Y \subset X$ un fermé. Soit $F$ un faisceau algébrique cohérent sur $X$ dont le support est $X$. Soit $n$ un entier. Les conditions suivantes sont équivalentes :

(i) pour tout point $x \in Y$, on a $\operatorname{prof}\left(F_{x}\right) \geq n$;

(ii) pour $i<n, \mathcal{H}_{Y}^{i}(F)=0$.

Cet énoncé entraîne que $\mathcal{H}_{Y}^{i}\left(\mathrm{M}_{u}, \omega\right)=0$ pour $i=0,1$. De la suite longue de cohomologie à support on déduit que $\omega=j_{*}\left(j^{*}(\omega)\right)$. Le même argument appliqué au faisceau inversible $\pi^{*} \mathcal{O}(-3 d)$ implique

$$
\pi^{*} \mathcal{O}(-3 d)=j_{*}\left(j^{*}\left(\pi^{*} \mathcal{O}(-3 d)\right)\right),
$$

donc il suffit de démontrer l'isomorphisme souhaité sur l'ouvert $\mathrm{M}_{u}^{s}$.

On note $\Omega^{s}$ la préimage de $\mathrm{M}_{u}^{s}$ par le morphisme $\rho: \Omega^{\mathrm{ss}} \rightarrow \mathrm{M}_{u}$.

Lemme 3.3. - L'application $\rho^{*}: \operatorname{Pic}\left(\mathrm{M}_{u}^{s}\right) \rightarrow \operatorname{Pic}\left(\Omega^{s}\right)$ est injective.

Preuve. - L'action de GL $(H)$ sur $\Omega^{s}$ se factorise à travers une action propre et libre du groupe $G=\operatorname{PGL}(H)$, et $\mathrm{M}_{u}^{s}$ est le quotient de cette action (cf. [17], lemme 2.4). En appliquant le lemme de descente de Kempf (th. 2.3, p. 63, et remarque p. 66, [8]), on obtient un isomorphisme $\mathrm{Pic}\left(\mathrm{M}_{u}^{s}\right)=\mathrm{Pic}^{G}\left(\Omega^{s}\right)$, où $\mathrm{Pic}^{G}$ désigne le groupe des fibrés inversibles munis d'une action de $G$. On a la suite exacte (voir [18], §3.3) :

$$
0 \rightarrow \mathrm{H}^{1}\left(G, \mathcal{O}^{*}\left(\Omega^{s}\right)\right) \longrightarrow \operatorname{Pic}^{G}\left(\Omega^{s}\right) \longrightarrow \operatorname{Pic}\left(\Omega^{s}\right)
$$

où $\mathrm{H}^{1}\left(G, \mathcal{O}^{*}\left(\Omega^{s}\right)\right)$ est l'espace des morphismes croisés $\phi: G \times \Omega^{s} \rightarrow \mathbb{C}^{*}$, c'està-dire qui vérifient

$$
\phi\left(g g^{\prime}, x\right)=\phi\left(g, g^{\prime} x\right) \cdot \phi\left(g^{\prime}, x\right) .
$$

Mais $G=\operatorname{PGL}(H)$ et les seules fonctions régulières inversibles sur $\operatorname{GL}(H)$ sont les caractères de $\mathrm{GL}(H)$, donc les seules fonctions régulières inversibles sur $\operatorname{PGL}(H)$ sont les constantes. Pour un morphisme croisé $\phi: G \times \Omega^{s} \rightarrow \mathbb{C}^{*}$ on a $\phi(e, x)=1$ et la fonction $\phi_{x}: G \rightarrow \mathbb{C}^{*}$ est régulière inversible. Cela implique que tout morphisme croisé est constant égal à 1 , et la conclusion.

Par conséquent, il suffit de montrer

$$
\rho^{*} \omega=\rho^{*} \pi^{*} \mathcal{O}(-3 d)
$$

dans $\operatorname{Pic}\left(\Omega^{s}\right)$. Il suffit encore de le prouver dans $\operatorname{Pic}\left(\Omega^{s}\right) \otimes \mathbb{Q}$, puisque $\operatorname{Pic}\left(\mathrm{M}_{u}\right)=$ $\operatorname{Pic}\left(\mathrm{M}_{u}^{s}\right)$ est sans torsion $(c f$. th. $3.5,[17])$. On démontre cette affirmation en 
appliquant la formule de Riemann-Roch-Grothendieck. Si $\mathbf{T}_{\mathrm{M}_{u}^{s}}$ est le fibré tangent à $\mathrm{M}_{u}^{s}$ et $\mathcal{G}$ est la famille universelle de faisceaux stables de dimension 1 paramétrée par $\Omega^{s}$, on a

$$
\rho^{*} \mathbf{T}_{\mathrm{M}_{u}^{s}}=\underline{\operatorname{Ext}}_{\mathrm{pr}_{1}}^{1}(\mathcal{G}, \mathcal{G}) .
$$

Puisque pour chaque $s \in \Omega^{s}, \mathcal{G}_{s}$ est un faisceau stable, on a $\operatorname{Hom}\left(\mathcal{G}_{s}, \mathcal{G}_{s}\right)=\mathbb{C}$ pour tout $s$, donc $\underline{\operatorname{Hom}}(\mathcal{G}, \mathcal{G})=\mathcal{O}$. Alors

$$
\underline{\operatorname{Ext}_{\mathrm{pr}_{1}}^{0}}(\mathcal{G}, \mathcal{G})=\operatorname{pr}_{1 *} \underline{\operatorname{Hom}}(\mathcal{G}, \mathcal{G})=\operatorname{pr}_{1 *} \mathcal{O}=\mathcal{O}_{\Omega^{s}} .
$$

Chaque faisceau $\mathcal{G}_{s}$ est de dimension 1 , donc on a aussi Ext $_{\text {pr }_{1}}^{i}(\mathcal{G}, \mathcal{G})=0$ pour $i \geq 2$. Alors

$$
\operatorname{det} \underline{\operatorname{Ext}}_{\mathrm{pr}_{1}}^{\bullet}(\mathcal{G}, \mathcal{G})=\left[\operatorname{det} \underline{\operatorname{Ext}}_{\mathrm{pr}_{1}}^{1}(\mathcal{G}, \mathcal{G})\right]^{-1} .
$$

On peut calculer

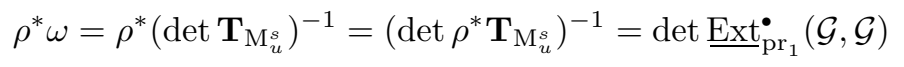

dans $\operatorname{Pic}\left(\Omega^{s}\right) \otimes \mathbb{Q}$ avec la formule de Riemann-Roch-Grothendieck :

$$
\rho^{*} \omega=\operatorname{pr}_{1 *}\left(\left[(\operatorname{ch} \mathcal{G})^{*}(\operatorname{ch} \mathcal{G}) \operatorname{Td}\left(\mathbb{P}_{2}\right)\right]_{3}\right) .
$$

On a

$$
\left[(\operatorname{ch} \mathcal{G})^{*}(\operatorname{ch} \mathcal{G}) \operatorname{Td}\left(\mathbb{P}_{2}\right)\right]_{3}=-\left(\operatorname{ch}_{1} \mathcal{G}\right)^{2} \operatorname{Td}_{1}\left(\mathbb{P}_{2}\right)=\frac{1}{2} c_{1}^{2}(\mathcal{G}) c_{1}\left(\omega_{\mathbb{P}_{2}}\right) .
$$

Calculons $c_{1}(\mathcal{G}) \in \operatorname{Pic}\left(\Omega^{s} \times \mathbb{P}_{2}\right)$. On a $c_{1}(\mathcal{G})=\rho^{*} \pi^{*} \mathcal{O}(1) \otimes \mathcal{O}(d)$ par le corollaire 4.2. On note $\mathfrak{h}=\rho^{*} \pi^{*} \mathcal{O}(1)$ et $h=\operatorname{pr}_{2}^{*} \mathcal{O}(1)$ dans $\operatorname{Pic}\left(\Omega^{s} \times \mathbb{P}_{2}\right)$. En notation additive on trouve $c_{1}(\mathcal{G})=\mathfrak{h}+d h$. On obtient dans $(10)$ :

$$
\rho^{*} \omega=\operatorname{pr}_{1 *}\left(\left[\frac{1}{2}(\mathfrak{h}+d h)^{2}(-3 h)\right]\right)=-3 d \mathfrak{h}=\rho^{*} \pi^{*} \mathcal{O}(-3 d)
$$

par la formule de projection. L'égalité (9) est prouvée, donc la proposition.

3.2. Le faisceau canonique et le fibré déterminant de Donaldson sur $\mathbf{M}_{\boldsymbol{c}}$. - Dans ce paragraphe $c$ désignera une classe générale $c=\left(r, c_{1}, \chi\right)$ dans $\mathrm{K}\left(\mathbb{P}_{2}\right)$ telle que $r>0$ et $\mathrm{M}_{c}$ soit non-vide. Le fibré $\mathcal{D}=\mathcal{D}_{\mathfrak{u}}$ sera le fibré déterminant de Donaldson associé à la classe orthogonale à $c, \mathfrak{u}=\left(0, r / \delta,-c_{1} / \delta\right)$ où $\delta=\operatorname{pgcd}\left(\mathrm{r}, \mathrm{c}_{1}\right)$.

Proposition 3.4. - Le faisceau dualisant $\omega_{\mathrm{M}_{c}}$ est inversible et dansPic $\left(\mathrm{M}_{c}\right)$, on $a$

$$
\omega_{\mathrm{M}_{c}} \simeq \mathcal{D}^{\otimes-3 \delta} .
$$

TOME $130-2002-\mathrm{N}^{\mathrm{O}} 1$ 
Preuve. - Soit $\mathrm{M}_{c}^{s}$ l'ouvert des classes représentant des faisceaux stables. Il y a deux cas à considérer : quand la codimension du complémentaire $C_{\mathrm{M}_{c}^{s}}$ de $\mathrm{M}_{c}^{s}$ dans $\mathrm{M}_{c}$ est $\geq 2$ et quand ce fermé est une hypersurface.

Dans le premier cas la démonstration est identique à celle de la proposition 3.1. On se ramène à prouver l'isomorphisme sur $\mathrm{M}_{c}^{s}$. Comme le groupe $\operatorname{Pic}\left(\mathrm{M}_{c}\right)=\operatorname{Pic}\left(\mathrm{M}_{c}^{s}\right)$ est sans torsion, il suffit de prouver l'isomorphisme dans $\operatorname{Pic}\left(\Omega^{s}\right) \otimes \mathbb{Q}$, où $\Omega^{s}$ est l'image réciproque de $\mathrm{M}_{c}^{s}$ dans $\Omega^{\mathrm{ss}}$. Les calculs pour les premières classes de Chern des fibrés $\rho^{*}\left(\omega_{\mathrm{M}_{c}}\right)$ et $\rho^{*}\left(\mathcal{D}^{\otimes-3 \delta}\right) \operatorname{dans} \operatorname{Pic}\left(\Omega^{s}\right) \otimes \mathbb{Q}$ ont été faits par O'Grady [25] en utilisant la formule de Riemann-Roch-Grothendieck. Puisque ces classes coïncident, le résultat découle.

Une analyse du second cas a été faite par Drézet [6]. Il est prouvé que la classe $c$ est divisible par 2, l'espace de modules $\mathrm{M}_{\frac{1}{2} c}$ s'identifie à $\mathbb{P}_{2}$, le fibré déterminant $\mathcal{D}_{\mathfrak{u}}$ sur $M_{\frac{1}{2} c}$ s'identifie à $\mathcal{O}_{\mathbb{P}_{2}}(1)$ et l'espace de modules $\mathrm{M}_{c}$ s'identifie à $\mathbb{P}_{5}$. L'hypersurface des points strictement semi-stables est l'image du morphisme

$$
\operatorname{Sym}^{2}\left(\mathrm{M}_{\frac{1}{2} c}\right) \longrightarrow \mathrm{M}_{c}
$$

qui associe aux classes $[E],[F]$ la classe $[E] \oplus[F]$ dans $\mathrm{M}_{c}$. Lorsque la classe $[F]$ est fixée, le morphisme

$$
\phi_{F}: \mathrm{M}_{\frac{1}{2} c}=\mathbb{P}_{2} \longrightarrow \mathrm{M}_{c}=\mathbb{P}_{5}
$$

qui associe à $[E]$ la classe $[E] \oplus[F]$ est linéaire.

Cela suffit pour terminer la preuve de la proposition. En effet, le fibré canonique sur $\mathbb{P}_{5}$ est $\omega_{\mathbb{P}_{5}}=\mathcal{O}_{\mathbb{P}_{5}}(-6)$ et il suffit de prouver que $\mathcal{D}_{\mathfrak{u}}$ s'identifie à $\mathcal{O}_{\mathbb{P}_{5}}(1)$. On prouve facilement que $\phi_{F}^{*}\left(\mathcal{D}_{\mathfrak{u}}\right)=\mathcal{D}_{\mathfrak{u}}$. Puisque $\phi_{F}$ est linéaire on a aussi $\phi_{F}^{*}\left(\mathcal{O}_{\mathbb{P}_{5}}(1)\right)=\mathcal{O}_{\mathbb{P}_{2}}(1)$. On avait vu que l'isomorphisme $\mathcal{D}_{\mathfrak{u}} \simeq \mathcal{O}_{\mathbb{P}_{2}}(1)$ était satisfait sur $\mathrm{M}_{\frac{1}{2} c}=\mathbb{P}_{2}$. Puisque l'application $\phi_{F}^{*}$ est bijective on obtient que $\mathcal{D}_{\mathfrak{u}}=\mathcal{O}(1)$ dans $\operatorname{Pic}\left(\mathrm{M}_{c}\right)$.

Remarque 3.5. - Le calcul du faisceau dualisant sur $\mathrm{M}_{c}$ a déjà été fait par Drézet à partir des monades, sans qu'il ait reconnu le rôle du fibré déterminant de Donaldson [7], th. F, et par O'Grady [25], sur l'ouvert des points stables. Le calcul fait dans la proposition 3.1 pour le faisceau dualisant de $\mathrm{M}_{d \mathfrak{u}}$ est calqué sur ce dernier.

DÉFInition 3.6 ( $c f$. [9], 5.5 (b), p. 45). - 1) Un fibré inversible $L$ sur une variété algébrique projective $X$ est dit nef si pour toutes les courbes $C \subset X$ on a $\operatorname{deg}\left(\left.L\right|_{C}\right) \geq 0$.

2) Un fibré inversible nef $L$ sur une variété algébrique projective irréductible $X$ est dit big si $\int_{X} c_{1}(L)^{\operatorname{dim} X}>0$.

Proposition 3.7. - Le fibré $\mathcal{D}$ est nef et big. 
Preuve. - D'après [16], [21], il existe un entier $k$ satisfaisant aux conditions suivantes :

- le fibré $\mathcal{D}^{\otimes k}$ est engendré par ses sections ;

- considérons le morphisme associé $\phi_{k}: \mathrm{M}_{c} \rightarrow \mathbb{P} \cdot \mathrm{H}^{0}\left(\mathrm{M}_{c}, \mathcal{D}^{\otimes k}\right)$ dans l'espace projectif des hyperplans de $\mathrm{H}^{0}\left(\mathrm{M}_{c}, \mathcal{D}^{\otimes k}\right)$; la restriction de $\phi_{k}$ à l'ouvert des fibrés $\mu$-stables est à fibres finies.

De la première condition on déduit que $\mathcal{D}$ est nef, de la seconde que le nombre $\int_{\mathrm{M}_{c}} c_{1}(\mathcal{D})^{\operatorname{dim} \mathrm{M}_{c}}$ est strictement positif, c'est-à-dire que $\mathcal{D}$ est big.

Par un théorème de Boutot [4], l'espace de modules $\mathrm{M}_{c}$ est à singularités rationnelles. Par ailleurs le théorème de Kawamata-Viehweg est valable sur les variétés à singularités rationnelles (voir [9], 5.12 (c), p. 49). Nous obtenons d'après les propositions 3.4 et 3.7 le théorème :

ThÉORÈme 3.8. - Pour $q>0$ et $k>-3 \delta$, on a $\mathrm{H}^{q}\left(\mathrm{M}_{c}, \mathcal{D}^{\otimes k}\right)=0$.

\section{Les espaces de modules $\mathrm{M}_{d \mathfrak{u}}$}

On considère la classe d'un point $\eta^{2}=(0,0,1) \in \mathrm{K}\left(\mathbb{P}_{2}\right)$. C'est une classe orthogonale à $u=d \mathfrak{u}$. Dans la suite, nous allons étudier les fibrés $\mathcal{D}_{c}$ sur $\mathrm{M}_{u}$, pour $\langle c, u\rangle=0$. On peut écrire $c$ sous la forme $r(c)[\mathcal{O}]-n \eta^{2} \in \mathrm{K}\left(\mathbb{P}_{2}\right)$. Par additivité, il suffit d'étudier $\mathcal{D}_{\eta^{2}}$ et $\mathcal{D}_{\mathcal{O}}$.

Proposition 4.1. - On a $\pi^{*} \mathcal{O}(1)=\mathcal{D}_{\eta^{2}}^{-1}$.

Preuve. - On note $\Xi$ l'hypersurface universelle dans $C_{d} \times \mathbb{P}_{2}$ paramétrée par $C_{d}$. La proposition 2.8 de [18] affirme que $\mathcal{D}_{\eta^{2}}^{-1}=\pi^{*}\left(\lambda_{\mathcal{O}_{\Xi}}\left(\eta^{2}\right)\right)$ où

$$
\lambda_{\mathcal{O}_{\Xi}}\left(\eta^{2}\right)=\operatorname{det} \operatorname{pr}_{1 !}\left(\mathcal{O}_{\Xi} \cdot \operatorname{pr}_{2}^{*}\left(\eta^{2}\right)\right) \text {. }
$$

En partant de la résolution de $\mathcal{O}_{\Xi}$ sur $C_{d} \times \mathbb{P}_{2}$ :

$$
0 \rightarrow \mathcal{O}(-1,-d) \longrightarrow \mathcal{O} \longrightarrow \mathcal{O}_{\Xi} \rightarrow 0
$$

on obtient $\lambda_{\mathcal{O}_{\Xi}}\left(\eta^{2}\right)=\mathcal{O}(1)$ sur $C_{d}$.

Soit $\mathcal{G}$ une famille de faisceaux $G$ de dimension 1 paramétrée par une variété algébrique intègre $S$, et

$$
\phi: S \longrightarrow \mathrm{M}_{d \mathfrak{u}}
$$

le morphisme modulaire associé.

Corollaire 4.2. - On a $\operatorname{det} \mathcal{G}=(\phi \circ \pi)^{*}(\mathcal{O}(1)) \otimes \mathcal{O}(d)$ sur $S \times \mathbb{P}_{2}$.

TOME $130-2002-\mathrm{N}^{\mathrm{O}} 1$ 
Preuve du corollaire. - On note $(\operatorname{det} \mathcal{G})_{s}$ la restriction $\operatorname{de} \operatorname{det} \mathcal{G}$ à $\{s\} \times \mathbb{P}_{2}$ et $(\operatorname{det} \mathcal{G})_{x}$ la restriction $\operatorname{de} \operatorname{det} \mathcal{G}$ à $S \times\{x\}$. D'après le lemme 2.10 il suffit de prouver que $(\operatorname{det} \mathcal{G})_{s}=\mathcal{O}(d)$ sur $\mathbb{P}_{2}$ et que $(\operatorname{det} \mathcal{G})_{x}=(\phi \circ \pi)^{*}(\mathcal{O}(1))$ sur $S$. On a évidemment $(\operatorname{det} \mathcal{G})_{s}=\mathcal{O}(d)$ car $\mathcal{G}$ est une famille de faisceaux de classe $c_{1}=d$ sur $\mathbb{P}_{2}$ paramétrée par $S$. Considérons la résolution (1) pour $\mathcal{G}$. On note $\mathcal{Q}_{x}$, $\mathcal{R}_{x}, \mathcal{G}_{x}$ les restrictions de $\mathcal{Q}, \mathcal{R}$, et respectivement $\mathcal{G}$ à $S \times\{x\}$. On a une suite exacte :

$$
0 \rightarrow \underline{\operatorname{Tor}}_{1}^{S \times \mathbb{P}_{2}}\left(\mathcal{G}, \operatorname{pr}_{2}^{*} \mathcal{O}_{x}\right) \longrightarrow \mathcal{Q}_{x} \longrightarrow \mathcal{R}_{x} \longrightarrow \mathcal{G}_{x} \rightarrow 0
$$

Chacun de ces faisceaux a un support inclus dans $S \times\{x\}$, donc leur image directe supérieure $R^{i} \mathrm{pr}_{1 *}$ est nulle pour $i>0$. Il résulte que

$$
\operatorname{pr}_{1 !}\left(\mathcal{G} \cdot \operatorname{pr}_{2}^{*} \mathcal{O}_{x}\right)=\left[\operatorname{pr}_{1 *}\left(\mathcal{G}_{x}\right)\right]-\left[\operatorname{pr}_{1 *}\left(\underline{\operatorname{Tor}}_{1}^{S \times \mathbb{P}_{2}}\left(\mathcal{G}, \operatorname{pr}_{2}^{*} \mathcal{O}_{x}\right)\right)\right]=\left[\mathcal{R}_{x}\right]-\left[\mathcal{Q}_{x}\right]
$$

où on a identifié $\mathcal{Q}_{x}$ avec $\operatorname{pr}_{1 *}\left(\mathcal{Q}_{x}\right)$ et $\mathcal{R}_{x}$ avec $\operatorname{pr}_{1 *}\left(\mathcal{R}_{x}\right)$. Par la définition de $\mathcal{D}_{\eta^{2}}^{-1}$ on trouve :

$$
\phi^{*} \mathcal{D}_{\eta^{2}}^{-1}=\phi^{*} \mathcal{D}_{\left[\mathcal{O}_{x}\right]}^{-1}=\left(\operatorname{det} \mathcal{Q}_{x}\right)^{-1} \otimes \operatorname{det} \mathcal{R}_{x} .
$$

D'après la proposition 4.1 il résulte que

$$
\begin{aligned}
(\operatorname{det} \mathcal{G})_{s} & =\left(\operatorname{det} \mathcal{Q}_{x}\right)^{-1} \otimes \operatorname{det} \mathcal{R}_{x} \\
& =\phi^{*} \mathcal{D}_{\eta^{2}}^{-1}=(\phi \circ \pi)^{*}(\mathcal{O}(1)) .
\end{aligned}
$$

On définit le fibré inversible $\Theta$ sur $\mathrm{M}_{u}$ comme $\Theta=\mathcal{D}_{\mathcal{O}}=\operatorname{det} \mathrm{pr}_{1 !}(u)^{-1}$. Tout ce qu'on utilisera dans la suite est contenu dans la proposition suivante, extraite de [17], chap. 2 :

Proposition 4.3 (voir [17]). - Le fibré $\Theta$ a une section canonique $\theta$, unique $\grave{a}$ constante près, non identiquement nulle, qui s'annule aux points $G$ tels que $h^{0}\left(\mathbb{P}_{2}, G\right)=h^{1}\left(\mathbb{P}_{2}, G\right) \neq 0$.

\subsection{Les cas $d=1,2$}

Proposition 4.4 (voir [17]). - Pour $d=1,2$, le morphisme $\pi$ est un isomorphisme, et le fibré $\Theta$ est trivial. En conclusion, les espaces $\mathbb{P H}^{0}\left(\mathrm{M}_{d \mathfrak{u}}, \Theta^{\otimes r}(n)\right)$ et $\mathbb{P H}^{0}\left(C_{d}, \mathcal{O}(n)\right)$ s'identifient.

Preuve. - Le fait que $\pi$ est un isomorphisme dans ce cas est démontré dans [17]. Plus précisément, l'inverse de $\pi$ est donné de la manière suivante : pour $d=1$, à une droite $\ell$ on associe le faisceau $\mathcal{O}_{\ell}(-1)$, pour $d=2$ et pour une conique $C$ lisse, à $C$ on associe le faisceau $\mathcal{O}_{C}(-a) \simeq \mathcal{O}_{\mathbb{P}_{1}}(-1)$ (a point de $C$ ), pour $d=2$ et $C$ décomposable en deux droites $\ell_{1}$ et $\ell_{2}$, à $C$ on associe le faisceau $\mathcal{O}_{\ell_{1}}(-1) \oplus \mathcal{O}_{\ell_{2}}(-1)$. Pour chacun de ces faisceaux on a $h^{0}=h^{1}=0$. Il résulte que la section $\theta$ est partout non nulle sur $\mathrm{M}_{d \mathfrak{u}}$. Donc le fibré $\Theta$ est trivial. La conclusion résulte de la proposition 4.1, du fait que $\pi$ est un isomorphisme et de la trivialité du fibré $\Theta$. 
On déduit en corollaire :

Corollaire 4.5. - Pour $c=2-n \eta^{2}$, on $a$ :

$$
\begin{array}{r}
\mathrm{H}^{0}\left(\mathrm{M}_{\mathfrak{u}}, \mathcal{D}_{c}\right)=\mathrm{H}^{0}\left(\mathbb{P}_{2}^{*}, \mathcal{O}_{\mathbb{P}_{2}^{*}}(n)\right)=\mathbf{S}^{n} E^{*}, \\
\mathrm{H}^{0}\left(\mathrm{M}_{2 \mathfrak{u}}, \mathcal{D}_{c}\right)=\mathrm{H}^{0}\left(\mathbb{P}_{5}, \mathcal{O}_{\mathbb{P}_{5}}(n)\right)=\mathbf{S}^{n}\left(\mathbf{S}^{2} E^{*}\right) .
\end{array}
$$

On rappelle que $E=\mathrm{H}^{0}\left(\mathbb{P}_{2}, \mathcal{O}(1)\right)$.

4.2. Le cas $\boldsymbol{d}=\mathbf{3}$. - Soit $\mathfrak{u}$ la classe $(0,1,0) \in \mathrm{K}\left(\mathbb{P}_{2}\right)$ et $c=\left(2,0, c_{2}=n\right)$. L'objectif de ce paragraphe est de démontrer la :

Proposition 4.6. - En tant que $\mathrm{SL}(3)$-représentation l'espace $\mathrm{H}^{0}\left(\mathrm{M}_{3 \mathfrak{u}}, \mathcal{D}_{c}\right)$ s'identifie à $\mathbf{S}^{n}\left(\mathbf{S}^{3} E^{*}\right) \oplus \mathbf{S}^{n-2}\left(\mathbf{S}^{3} E^{*}\right)$ ( où $E=\mathrm{H}^{0}\left(\mathbb{P}_{2}, \mathcal{O}(1)\right)$ ).

Notre référence principale sera l'article [17]. On considère la cubique universelle $\mathcal{C} \subset C_{3} \times \mathbb{P}_{2}$. La projection $\mathcal{C} \rightarrow C_{3}$ induit par la propriété universelle de l'espace de modules $\mathrm{M}_{3 \mathfrak{u}}$ un morphisme $s: C_{3} \rightarrow \mathrm{M}_{3 \mathfrak{u}}$ qui associe à une cubique $C$ son faisceau structural $\mathcal{O}_{C}$. Le morphisme $s$ est une section de $\pi$ : la résolution

$$
0 \rightarrow \mathcal{O}(-3) \stackrel{\cdot C}{\longrightarrow} \mathcal{O} \longrightarrow \mathcal{O}_{C} \rightarrow 0
$$

démontre que $(\pi \circ s)(C)=C$. Ici $\underline{C}$ désigne l'équation de la cubique $C$.

Prenons $D \subset \mathbb{P}_{2} \times \mathbb{P}_{2}^{*}$ la variété d'incidence et $p$ et $q$ les projections :

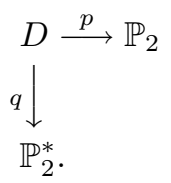

L'application $G \rightarrow q_{*}\left(p^{*}(G(-2))\right)(-1)$ définit (cf. [17]) un morphisme $\phi$ : $\mathrm{M}_{3 \mathfrak{u}} \rightarrow \mathrm{M}_{(3,0,0)}^{*}$ dans l'espace de modules de faisceaux semi-stables de classe $\left(r=3, c_{1}=0, \chi=0\right)$ sur $\mathbb{P}_{2}^{*}$. On note encore $\mathcal{D}=\mathcal{D}_{u}$ le fibré déterminant sur $\mathrm{M}_{(3,0,0)}^{*}$, associé à la classe $\mathfrak{u}=(0,1,0)$. La proposition suivante est prouvée dans [17] :

Proposition 4.7. - (i) Le diviseur des zéros div $\theta$ de la section $\theta$ coüncide avec l'image du morphisme s.

(ii) Le morphisme $\phi$ est l'éclatement d'un point lisse et la fibre exceptionnelle est $\operatorname{div} \theta$.

On obtient que les morphismes $\pi, s$ établissent un isomorphisme entre div $\theta$ et $C_{3}$. On identifiera $\operatorname{div} \theta$ et $C_{3}$ dans la suite. Puisque $\operatorname{div} \theta$ est le diviseur exceptionnel, son fibré conormal est $\mathcal{O}_{C_{3}}(1)$. De la suite exacte courte

$$
0 \rightarrow \Theta^{-1} \stackrel{\theta}{\longrightarrow} \mathcal{O} \longrightarrow \mathcal{O}_{\operatorname{div} \theta} \rightarrow 0
$$

TOME $130-2002-\mathrm{N}^{\mathrm{O}} 1$ 
il résulte que

$$
\left.\Theta^{-1}\right|_{\operatorname{div} \theta} \simeq \mathcal{O}_{C_{3}}(1) \text {. }
$$

Proposition 4.8. - On a $\Theta(1)=\phi^{*} \mathcal{D}$ dans $\operatorname{Pic}\left(\mathrm{M}_{3 \mathfrak{u}}\right)$.

Preuve. - Compte-tenu du fait que Pic $\left(\mathrm{M}_{3 \mathfrak{u}}\right)$ est sans torsion ( $c f$. [17], th. 3.5), il suffit de démontrer que

$$
\phi^{*} \mathcal{D}^{\otimes-9}=\Theta^{\otimes-9}(-9) .
$$

Par la proposition 3.4 , on a $\mathcal{D}^{\otimes-9}=\omega_{\mathrm{M}_{(3,0,0)}^{*}}$, et par la proposition 3.1 , on a $\mathcal{O}(-9)=\omega_{\mathrm{M}_{3 \mathfrak{u}}}$. L'égalité à démontrer devient

$$
\phi^{*} \omega_{M_{(3,0,0)}^{*}}=\omega_{M_{3 u}} \otimes \Theta^{\otimes-9} .
$$

Mais on a vu que $\Theta=\mathcal{O}(\operatorname{div} \theta)$ et que $\operatorname{div} \theta$ est le diviseur exceptionnel. L'égalité à démontrer est

$$
\omega_{\mathrm{M}_{3 \mathfrak{u}}}=\phi^{*} \omega_{\mathrm{M}_{(3,0,0)}^{*}} \otimes \mathcal{O}(9 \operatorname{div} \theta)
$$

qui est valable chaque fois qu'on éclate un point lisse dans une variété de dimension 10 (voir [12], ex. II 8.5, p. 188).

Puisque $c=2-n \eta^{2}$ dans $\mathrm{K}\left(\mathbb{P}_{2}\right)$, on a $\mathcal{D}_{c} \simeq \Theta^{\otimes 2}(n)$ sur $\mathrm{M}_{3 \mathfrak{u}}$. On passe au calcul de l'espace $\mathrm{H}^{0}\left(\mathrm{M}_{3 \mathfrak{u}}, \Theta^{\otimes 2}(n)\right)$.

Proposition 4.9. - $\mathrm{H}^{q}\left(\mathrm{M}_{3 \mathfrak{u}}, \Theta(n)\right)=0$ pour $q \geq 1$ et $n \geq-8$.

Preuve. - L'espace de modules $\mathrm{M}_{3 \mathfrak{u}}$ est le quotient d'une variété lisse par un groupe réductif, donc il est à singularités rationnelles, par un résultat de Boutot [4]. Le théorème de Kawamata-Viehweg s'applique (voir [9], 5.12 (c), p. 49). D'après la proposition 3.7 le fibré $\mathcal{D}$ est nef et big sur $\mathrm{M}_{(3,0,0)}^{*}$ et d'après les propositions 4.7 et 4.8 le fibré $\Theta(1)$ est l'image réciproque de $\mathcal{D}$ par un éclatement. On en déduit que le fibré $\Theta(1)$ est big et nef sur $M_{3 \mathfrak{u}}$. Le fibré $\mathcal{O}(n-1)=\pi^{*}(\mathcal{O}(n-1))$ est globalement engendré pour $n \geq 1$ donc $\Theta(n)$ est big et nef pour $n \geq 1$. La proposition 3.1 fournit le faisceau dualisant sur $\mathrm{M}_{d \mathfrak{u}}$ : $\omega_{\mathrm{M}_{d \mathfrak{u}}}=\pi^{*}(\mathcal{O}(-3 \bar{d}))$. Alors $\Theta(n) \otimes \omega_{\mathrm{M}_{d \mathfrak{u}}}^{-1}=\Theta(n+9)$ est big et nef pour $n \geq-8$. Le résultat en découle.

On tensorise la suite (12) par $\Theta^{\otimes 2}(n)$. On obtient, après l'identification $\operatorname{div} \theta=C_{3}$, et en utilisant l'isomorphisme (13), la suite exacte courte sur $\mathrm{M}_{3 \mathfrak{u}}$ :

$$
0 \rightarrow \Theta(n) \rightarrow \Theta^{\otimes 2}(n) \rightarrow \mathcal{O}_{C_{3}}(n-2) \rightarrow 0 .
$$

La proposition 4.9 conduit à une suite exacte courte sur les sections globales, pour $n \geq-8$ :

$$
\begin{aligned}
0 \rightarrow \mathrm{H}^{0}\left(\mathrm{M}_{3 \mathfrak{u}}, \Theta(n)\right) & \longrightarrow \mathrm{H}^{0}\left(\mathrm{M}_{3 \mathfrak{u}}, \Theta^{\otimes 2}(n)\right) \\
& \longrightarrow \mathrm{H}^{0}\left(C_{3}, \mathcal{O}_{C_{3}}(n-2)\right) \rightarrow 0 .
\end{aligned}
$$


Proposition 4.10. - Soit $u=3 \mathfrak{u}$. Alors les morphismes

$$
\mathrm{H}^{0}\left(C_{3}, \mathcal{O}(n)\right) \stackrel{\pi^{*}}{\longrightarrow} \mathrm{H}^{0}\left(\mathrm{M}_{u}, \mathcal{O}(n)\right) \stackrel{\cdot \theta}{\longrightarrow} \mathrm{H}^{0}\left(\mathrm{M}_{u}, \Theta(n)\right)
$$

sont des isomorphismes.

Preuve. - On considère l'ouvert $U \subseteq C_{3}$ des courbes irréductibles. Son complémentaire $C_{U}$ est de codimension $\geq 2$. La proposition résulte des lemmes suivants :

LEMME 4.11. - Le complémentaire de l'image réciproque $\pi^{-1}(U)$ de l'ouvert $U$ par le morphisme $\pi$ est de codimension au moins 2 dans $\mathrm{M}_{u}$. En plus on a l'isomorphisme $\pi_{*}\left(\mathcal{O}_{\pi^{-1}(U)}\right)=\mathcal{O}_{U}$.

LEMME 4.12. - Le morphisme $\pi_{*} \mathcal{O}_{\mathrm{M}_{u}} \stackrel{\cdot \theta}{\longrightarrow} \pi_{*} \Theta$ est un isomorphisme sur $C_{3}$.

Effectivement, le lemme 4.11 implique :

$$
\begin{gathered}
\mathrm{H}^{0}\left(\mathrm{M}_{u}, \mathcal{O}(n)\right)=\mathrm{H}^{0}\left(\pi^{-1}(U), \mathcal{O}(n)\right)=\mathrm{H}^{0}\left(U, \pi_{*}(\mathcal{O}(n))\right) \\
=\mathrm{H}^{0}(U, \mathcal{O}(n))=\mathrm{H}^{0}\left(C_{3}, \mathcal{O}(n)\right) .
\end{gathered}
$$

Il résulte du lemme 4.12 que $\pi_{*} \mathcal{O}(n) \stackrel{\cdot \theta}{\longrightarrow} \pi_{*} \Theta(n)$ est un isomorphisme. En prenant les sections globales sur $C_{3}$ on obtient que $\mathrm{H}^{0}\left(\mathrm{M}_{u}, \mathcal{O}(n)\right) \stackrel{\cdot \theta}{\longrightarrow}$ $\mathrm{H}^{0}\left(\mathrm{M}_{u}, \Theta(n)\right)$ est un isomorphisme.

Preuve du lemme 4.11. - Soit $V \subseteq \mathrm{M}_{u}$ l'ouvert des faisceaux stables et localement libres sur leur support. Le lemme 3.2 et la proposition 3.4 de [17] démontrent que codim $C_{V} \geq 2$, où $C_{V}$ désigne le complémentaire de $V$. La proposition 2.8 de [17] affirme que le morphisme $V \stackrel{\pi}{\longrightarrow} C_{3}$ est lisse. Il résulte que ses fibres au-dessus de $C_{U}$ sont de dimension $\operatorname{dim} \mathrm{M}_{u}-\operatorname{dim} C_{3}=1$. L'inclusion $\pi^{-1}\left(C_{U}\right) \subset C_{V} \cup\left(V \cap \pi^{-1}\left(C_{U}\right)\right)$ entraîne codim $\pi^{-1}\left(C_{U}\right) \geq 2$. Le théorème 9 de [1] nous assure que le morphisme projectif $\pi^{-1}(U) \rightarrow U$ est plat et à fibres intègres. D'où $\pi_{*}\left(\mathcal{O}_{\pi^{-1}(U)}\right)=\mathcal{O}_{U}$.

Preuve du lemme 4.12. - On déduit de la suite exacte (12) et de l'isomorphisme (13) la suite exacte courte :

$$
0 \rightarrow \mathcal{O} \stackrel{\cdot \theta}{\longrightarrow} \Theta \rightarrow \mathcal{O}_{C_{3}}(-1) \rightarrow 0
$$

sur $\mathrm{M}_{3 \mathfrak{u}}$. On applique le foncteur $\pi_{*}$. On obtient sur $C_{3}$ la suite exacte :

$$
0 \rightarrow \pi_{*}(\mathcal{O}) \rightarrow \pi_{*}(\Theta) \stackrel{\cdot \theta}{\longrightarrow} \mathcal{O}_{C_{3}}(-1) \stackrel{\delta}{\longrightarrow} R^{1} \pi_{*}(\mathcal{O})
$$

Soit $W \subset C_{3}$ l'ouvert des cubiques lisses. La fibre $P_{C}$ du morphisme $\pi$ au-dessus de $C \in W$ s'identifie à la jacobienne $\operatorname{Jac}(C)$ de $C$. La restriction du fibré $\Theta$ à $P_{C}$ s'identifie au fibré $\Theta$ usuel sur Jac $(C)$. Alors le morphisme

TOME $130-2002-\mathrm{N}^{\mathrm{O}} 1$ 
$\mathrm{H}^{0}\left(P_{C}, \mathcal{O}\right) \rightarrow \mathrm{H}^{0}\left(P_{C}, \Theta\right)$ est un isomorphisme entre des espaces de dimension 1 . Puisque la fibration

$$
\pi^{-1}(W) \longrightarrow W
$$

est plate, par le théorème de semi-continuité, le morphisme $\pi_{*} \mathcal{O} \rightarrow \pi_{*}(\Theta)$ est un isomorphisme de fibrés inversibles sur $W$.

Il résulte de la suite exacte $(15)$ que le morphisme $\mathcal{O}_{C_{3}}(-1) \stackrel{\delta}{\rightarrow} R^{1} \pi_{*}(\mathcal{O})$ est injectif sur $W$. Comme $\mathcal{O}_{C_{3}}(-1)$ est un faisceau inversible, le morphisme $\delta$ est injectif partout sur $C_{3}$. Par conséquent le morphisme $\pi_{*} \mathcal{O}_{\mathrm{M}_{u}} \stackrel{\cdot \theta}{\rightarrow} \pi_{*} \Theta$ est un isomorphisme partout sur $C_{3}$.

D'après la proposition 4.10 et de la suite exacte (14) on obtient le

Corollaire 4.13. - Pour $n \geq-8$, on a une suite exacte courte

$$
0 \rightarrow \mathrm{H}^{0}\left(C_{3}, \mathcal{O}(n)\right) \stackrel{\theta^{2} \cdot \pi^{*}}{\longrightarrow} \mathrm{H}^{0}\left(\mathrm{M}_{3 \mathfrak{u}}, \Theta^{\otimes 2}(n)\right) \stackrel{s^{*}}{\longrightarrow} \mathrm{H}^{0}\left(C_{3}, \mathcal{O}(n-2)\right) \rightarrow 0 .
$$

D'où la proposition 4.6 .

\section{Injectivité du morphisme $\mathbf{D}_{c, u}$}

On commence par regarder le cas où la classe $c$ est de rang 1 . On utilise ensuite un argument de récurrence pour étendre le résultat au cas qui nous intéresse, où $c$ est de rang 2. Le début de la récurrence utilise le cas $c=$ $\left(1,0, c_{2}=n\right)$ étudié en préalable.

\subsection{Le cas $c=\left(1,0, c_{2}=n\right), u=d \mathfrak{u}$}

Proposition 5.1. - Pour $d=1,2,3$ et $n \geq 0$, l'image du morphisme $\Phi$ : $\mathrm{M}_{c} \rightarrow \mathbb{P H}^{0}\left(\mathrm{M}_{d \mathfrak{u}}, \mathcal{D}_{c}\right)$ n'est pas contenue dans un hyperplan.

On a vu que le morphisme

$$
\mathrm{H}^{0}\left(C_{d}, \mathcal{O}(n)\right) \stackrel{\pi^{*}}{\longrightarrow} \mathrm{H}^{0}\left(\mathrm{M}_{u}, \mathcal{O}(n)\right) \stackrel{\cdot \theta}{\longrightarrow} \mathrm{H}^{0}\left(\mathrm{M}_{u}, \Theta(n)\right)
$$

était bijectif pour $d=1,2,3$. Le lemme suivant sera utile :

LEMME 5.2. - Si $F=I_{Z}$ est l'idéal du sous-schéma $Z$ des $n$ points distincts $a_{1}, \ldots, a_{n}$ de $\mathbb{P}_{2}$, si $[G] \in \mathrm{M}_{d \mathfrak{u}}$, et s'il existe un point $a_{k} \in \operatorname{supp} G$, alors

$$
h^{0}(F \otimes G)=h^{1}(F \otimes G) \neq 0 .
$$

BULlETiN DE LA SOCIÉtÉ MATHÉMATIQUE DE FRANCE 
Preuve. - Sans restreindre la généralité, on peut supposer $a_{1}, \ldots, a_{i} \in \operatorname{supp} G$, $a_{i+1}, \ldots, a_{n} \notin \operatorname{supp} G$, pour un nombre $i \in\{1, \ldots, n\}$. On tensorise par $G$ la suite exacte :

$$
0 \rightarrow F \longrightarrow I_{\widehat{Z}} \longrightarrow \bigoplus_{j=1}^{i} \mathcal{O}_{a_{j}} \rightarrow 0
$$

où $I_{\widehat{Z}}$ désigne l'idéal du sous-schéma des $n-i$ points distincts $a_{i+1}, \ldots, a_{n}$ et $\mathcal{O}_{a_{j}}$ le faisceau structural du point $a_{j}$. En utilisant $\underline{\operatorname{Tor}}_{1}\left(I_{\widehat{Z}}, G\right)=0$ (puisque $I_{\widehat{Z}}$ est trivial au voisinage du support de $G$ ), on obtient une inclusion

$$
0 \rightarrow \underline{\mathrm{Tor}}_{1}\left(G, \mathcal{O}_{a_{i}}\right) \longrightarrow F \otimes G .
$$

Mais à partir de la résolution de longueur 1 de $G$ par des faisceaux localement libres $A$ et $B$ :

$$
0 \rightarrow A \stackrel{\alpha}{\longrightarrow} B \longrightarrow G \rightarrow 0
$$

on obtient après tensorisation par $\mathcal{O}_{a_{i}}$ :

$$
0 \rightarrow \underline{\operatorname{Tor}}_{1}\left(G, \mathcal{O}_{a_{i}}\right) \longrightarrow A_{\mid a_{i}} \stackrel{\left.\alpha\right|_{a_{i}}}{\longrightarrow} B_{\mid a_{i}} \longrightarrow G \otimes \mathcal{O}_{a_{i}} \rightarrow 0
$$

et det $\alpha$ est l'équation du support de $G \operatorname{donc} \operatorname{det} \alpha_{\left.\right|_{a_{i}}}=0$ et $\underline{\operatorname{Tor}}_{1}\left(G, \mathcal{O}_{a_{i}}\right) \neq 0$. Le faisceau $\underline{\operatorname{Tor}}_{1}\left(G, \mathcal{O}_{a_{i}}\right)$ a pour support le point $a_{i}$, donc $\mathrm{H}^{0}\left(\underline{\operatorname{Tor}}_{1}\left(G, \mathcal{O}_{a_{i}}\right)\right) \neq 0$, d'où $\mathrm{H}^{0}(F \otimes G) \neq 0$.

On introduit quelques notations. Pour $E=\mathrm{H}^{0}\left(\mathbb{P}_{2}, \mathcal{O}(1)\right)$, le point $a_{i}$ est un élément de $\mathbb{P}\left(E^{*}\right)$. Alors $a_{i}^{d}$ est un élément de $\mathbb{P}\left(\mathbf{S}^{d} E^{*}\right)$ et il représente, à une constante près, un élément dans $\mathrm{H}^{0}\left(C_{d}, \mathcal{O}(1)\right)$. C'est l'équation $H_{a_{i}}$ de l'hyperplan des courbes de degré $d$ qui passent par le point $a_{i} \in \mathbb{P}_{2}$. Alors $H_{a_{1}} \cdots H_{a_{n}} \in \mathbf{S}^{n} \mathrm{H}^{0}\left(C_{d}, \mathcal{O}(1)\right)=\mathrm{H}^{0}\left(C_{d}, \mathcal{O}(n)\right)$.

Lemme 5.3. - Pour $F=I_{Z}$ comme dans le lemme 5.2, on a $\sigma_{F}=\sigma_{c, u}(F)=$ $\operatorname{cst} \cdot \theta \cdot \pi^{*}\left(H_{a_{1}} \cdots \cdot H_{a_{n}}\right)$, pour une constante cst $\in \mathbb{C}^{*}$.

Ce lemme suffit pour démontrer la proposition 5.1, puisque $\left\{H_{a_{i}}\right\}_{i}$ engendrent $\mathrm{H}^{0}\left(C_{d}, \mathcal{O}(1)\right)$ et les produits des $\left\{H_{a_{i}}\right\}_{i}$ engendrent $\mathbf{S}^{n} \mathrm{H}^{0}\left(C_{d}, \mathcal{O}(1)\right)$.

Preuve du lemme 5.3. - Le lemme 5.2 nous dit que $\sigma_{F}$ s'annule sur tous les faisceaux $G$ dont le support contient le point $a_{i}$. Ces faisceaux appartiennent à l'ensemble d'équation $\pi^{*}\left(H_{a_{i}}\right)=0$. La section $\alpha=\sigma_{F} / \prod_{i=1}^{n} \pi^{*}\left(H_{a_{i}}\right)$ est une section rationnelle du fibré $\Theta$ sur $\mathrm{M}_{d \mathfrak{u}}$. Puisque $\sigma_{F}$ s'annule sur $\pi^{-1}\left(\left\{H_{a_{i}}=0\right\}\right)$, $\alpha$ est une section régulière. La proposition 4.10 appliquée pour $n=0$ nous assure que $\mathrm{H}^{0}\left(\mathrm{M}_{d \mathfrak{u}}, \Theta\right)=\mathrm{H}^{0}\left(\mathrm{M}_{d \mathfrak{u}}, \mathcal{O}\right)=\mathbb{C}$. Donc $\alpha=\operatorname{cst} \cdot \theta$.

On remarque, au passage, que la dualité étrange dans le cas $r^{c}=1, d=1,2,3$ a été prouvée :

Proposition 5.4. - Le morphisme de dualité étrange est un isomorphisme dans le cas $c=(1,0,1-n), u=(0, d, 0)$, pour $d=1,2,3$ et pour un entier positif $n$. 
Preuve. - En effet, le premier membre de la dualité est $\mathrm{H}^{0}\left(\mathrm{M}_{d \mathfrak{u}}, \Theta(n)\right)^{*}$, isomorphe par la proposition 4.10 à

$$
\mathrm{H}^{0}\left(C_{d}, \mathcal{O}(n)\right)^{*}=\mathbf{S}^{n}\left(\mathbf{S}^{d} E\right) .
$$

Pour identifier le second membre on utilise le fait que $\mathrm{M}_{\left(1,0, c_{2}=n\right)}$ coïncide avec le schéma de Hilbert $\operatorname{Hilb}^{n}\left(\mathbb{P}_{2}\right)$ des sous-schémas finis de longueur $n$ de $\mathbb{P}_{2}$.

Soit $\mathbf{S}^{n}\left(\mathbb{P}_{2}\right)$ le quotient de la puissance $n$-ième $\mathbb{P}_{2}^{n}$ de $\mathbb{P}_{2}$ par le groupe symétrique $\mathfrak{S}_{n}$. On dispose du morphisme de Hilbert-Chow

$$
H C: \operatorname{Hilb}^{n}\left(\mathbb{P}_{2}\right) \longrightarrow \mathbf{S}^{n}\left(\mathbb{P}_{2}\right)
$$

qui associe à un schéma fini $Z$ le cycle $\sum_{x \in \mathbb{P}_{2}} \lg Z_{x} x$. On note $\mathcal{O}(1,1, \ldots, 1)^{\mathfrak{S}_{n}}$ le quotient du fibré $\mathcal{O}(1,1, \ldots, 1)$ par l'action de $\mathfrak{S}_{n}$.

Lemme 5.5. - Les fibrés inversibles $\mathcal{D}_{\mathfrak{u}}$ et $H C^{*}\left(\mathcal{O}(1,1, \ldots, 1)^{\mathfrak{S}_{n}}\right)$ sont isomorphes sur $\operatorname{Hilb}^{n}\left(\mathbb{P}_{2}\right)$.

Preuve. - On considère le diagramme :

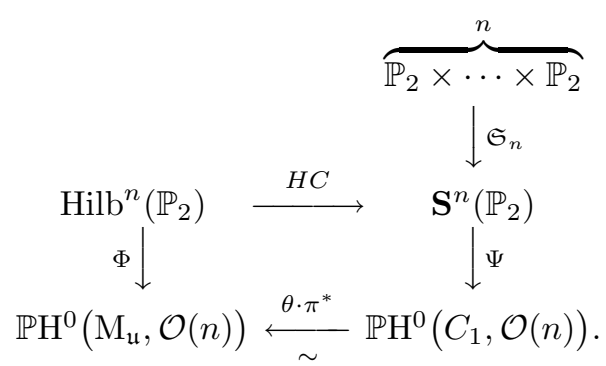

Ici, $C_{1}=\mathbb{P}_{2}^{*}$. Le morphisme $\Psi$ est défini par $\Psi\left(a_{1}, \ldots, a_{n}\right)=\left[H_{a_{1}} \cdots H_{a_{n}}\right]$. Le lemme 5.3 prouve que

$$
\Phi=\left(\theta \cdot \pi^{*}\right) \circ \Psi \circ H C
$$

sur l'ouvert des points distincts de $\operatorname{Hilb}^{n}\left(\mathbb{P}_{2}\right)$. Puisque cet ouvert est dense, le diagramme considéré est commutatif.

L'image réciproque du fibré $\mathcal{O}(1)$ de l'espace projectif $\mathbb{P H}^{0}\left(C_{1}, \mathcal{O}(n)\right)$, par $\Psi \circ \mathfrak{S}_{n}$, est le fibré $\mathcal{O}(1,1, \ldots, 1)$ sur $\mathbb{P}_{2}^{n}$ (on peut le vérifier sur chaque composante). On tient compte de l'isomorphisme (cf. [18], §3.4) :

$$
\operatorname{Pic}\left(\mathbf{S}^{n}\left(\mathbb{P}_{2}\right)\right) \longrightarrow \operatorname{Pic}\left(\mathbb{P}_{2}^{n}\right)^{\mathfrak{S}_{n}}
$$

On obtient que l'image réciproque du fibré $\mathcal{O}(1)$ de $\mathbb{P} H^{0}\left(C_{1}, \mathcal{O}(n)\right)$ sur $\mathbf{S}^{n}\left(\mathbb{P}_{2}\right)$ est $\mathcal{O}(1,1, \ldots, 1)^{\mathfrak{S}_{n}}$. Par la commutativité du diagramme on obtient la conclusion.

En passant aux puissances tensorielles supérieures, on trouve

$$
\mathcal{D}_{\mathfrak{u}}{ }^{\otimes d}=H C^{*}\left(\mathcal{O}(d, d, \ldots, d)^{\mathfrak{S}_{n}}\right) .
$$

BULLETIN DE LA SOCIÉTÉ MATHÉMATIQUe DE FRANCE 
Cela entraîne que $\mathrm{H}^{0}\left(\operatorname{Hilb}^{n}\left(\mathbb{P}_{2}\right), \mathcal{D}_{\mathfrak{u}}{ }^{\otimes d}\right)=\mathbf{S}^{n}\left(\mathbf{S}^{d} E\right)$. L'injectivité de $\mathrm{D}_{c, u}$ a été prouvée dans la proposition 5.1.

5.2. Le cas $c=\left(2,0, c_{2}=n\right), u=d \mathfrak{u}$

Proposition 5.6. - Pour $1 \leq d \leq 3$ et pour $n \geq 2$, l'image du morphisme $\Phi: \mathrm{M}_{c} \rightarrow \mathbb{P H}^{0}\left(\mathrm{M}_{d \mathfrak{u}}, \mathcal{D}_{c}\right)$ n'est pas contenue dans un hyperplan.

La proposition résulte des quatre lemmes suivants :

Lemme 5.7. - La proposition est vraie pour $n=2$.

LEMme 5.8. - L'application suivante est surjective :

$$
\begin{aligned}
\mathrm{H}^{0}\left(\mathrm{M}_{d \mathfrak{u}}, \Theta^{\otimes 2}(n)\right) \otimes \mathrm{H}^{0}\left(C_{d}, \mathcal{O}(1)\right) & \longrightarrow \mathrm{H}^{0}\left(\mathrm{M}_{d \mathfrak{u}}, \Theta^{\otimes 2}(n+1)\right), \\
s \otimes t & \longmapsto s \cdot \pi^{*} t .
\end{aligned}
$$

Lemme 5.9. - Soit $n \geq 2$ et $\mathrm{M}_{c}^{0} \subset \mathrm{M}_{c}$ l'ouvert des points stables. Si l'image $\Phi\left(\mathrm{M}_{c}\right)$ n'est pas contenue dans un hyperplan, alors $\Phi\left(\mathrm{M}_{c}{ }^{0}\right)$ n'est pas contenue dans un hyperplan.

Lemme 5.10. - Soit $F \in \mathrm{M}_{\left(2,0, c_{2}=n\right)}^{0}, x \in \mathbb{P}_{2}$ et a $: F \rightarrow \mathcal{O}_{x}$ un morphisme surjectif. Alors $F^{\prime}=\operatorname{Ker}$ a est un faisceau semi-stable et on a $\sigma_{F^{\prime}}=\sigma_{F} \cdot \pi^{*} H_{x}$ par l'application id $\cdot \pi^{*}$ du lemme 5.8. Ici

$\sigma_{F^{\prime}} \in \mathrm{H}^{0}\left(\mathrm{M}_{d \mathfrak{u}}, \Theta^{\otimes 2}(n+1)\right), \quad \sigma_{F} \in \mathrm{H}^{0}\left(\mathrm{M}_{d \mathfrak{u}}, \Theta^{\otimes 2}(n)\right)$ et $H_{x} \in \mathrm{H}^{0}\left(C_{d}, \mathcal{O}(1)\right)$.

Les lemmes 5.7, 5.8, 5.9 et 5.10 fournissent une démonstration par récurrence de la proposition 5.6. Le lemme 5.9 dit que les $\sigma_{F}$, pour $F$ stable, engendrent $\mathrm{H}^{0}\left(\mathrm{M}_{d \mathfrak{u}}, \Theta^{\otimes 2}(n)\right)$. Mais les $H_{x}$ engendrent $\mathrm{H}^{0}\left(C_{d}, \mathcal{O}(1)\right)$ lorsque $x$ varie. Par le lemme 5.8, on obtient que les $\sigma_{F} \cdot \pi^{*} H_{x}$ engendrent $\mathrm{H}^{0}\left(\mathrm{M}_{d \mathfrak{u}}, \Theta^{\otimes 2}(n+1)\right)$. Le lemme 5.10 assure que de tels éléments sont de la forme $\sigma_{F^{\prime}}$, donc des images par $\Phi$ de $\mathrm{M}_{\left(2,0, c_{2}=n+1\right)}$.

Preuve du lemme 5.8. - Pour $d=1,2$, le diagramme suivant est commutatif :

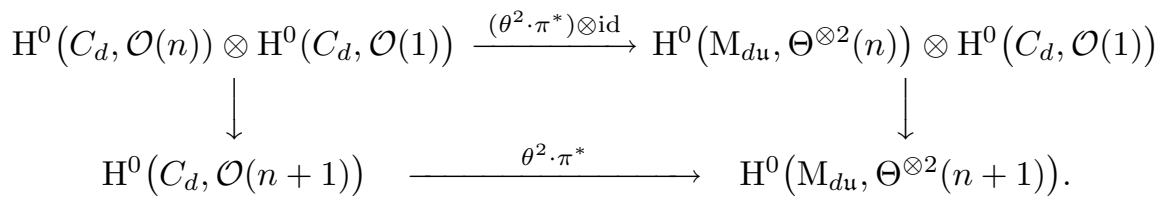

Par la proposition 4.4, les morphismes horizontaux sont des isomorphismes. Le lemme résulte de la surjectivité du morphisme vertical gauche.

TOME $130-2002-\mathrm{N}^{\mathrm{O}} 1$ 
Pour $d=3$, on note $H=\mathrm{H}^{0}\left(C_{d}, \mathcal{O}(1)\right)$. Le lemme est une conséquence $\mathrm{du}$ diagramme analogue, commutatif :

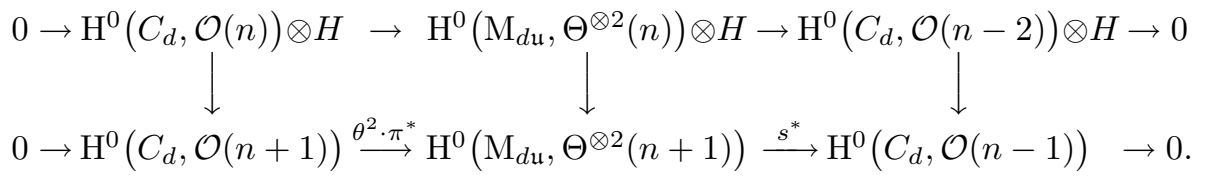

Les suites horizontales sont exactes d'après le corollaire 4.13. Les morphismes verticaux latéraux sont surjectifs, donc aussi le morphisme vertical central.

Preuve du lemme 5.9. - Ceci est évident, puisque $\mathrm{M}_{c}^{0}$ est un ouvert dense de $\mathrm{M}_{c}$.

Preuve du lemme 5.10:- On a $c_{1}\left(F^{\prime \prime}\right) \leq 0$ pour un sous-faisceau $F^{\prime \prime}$ de rang 1 de $F^{\prime}$ puisque $F^{\prime \prime}$ est aussi un sous-faisceau de $F$, et $F$ est stable. Si $c_{1}\left(F^{\prime \prime}\right)=0$ alors $\chi\left(F^{\prime \prime}\right)<\frac{1}{2}(2-n)-\frac{1}{2}=\frac{1}{2}(2-(n+1))$. Donc $F^{\prime}$ est semi-stable.

On tensorise par $G$ la suite exacte

$$
0 \rightarrow F^{\prime} \longrightarrow F \longrightarrow \mathcal{O}_{x} \rightarrow 0 .
$$

Soit $[G] \in \mathrm{M}_{d \mathfrak{u}}$. En tenant compte du lemme 2.3 (c), on obtient une suite exacte

$$
0 \longrightarrow \underline{\operatorname{Tor}}_{1}\left(G, \mathcal{O}_{x}\right) \longrightarrow F^{\prime} \otimes G \longrightarrow F \otimes G \longrightarrow G \otimes \mathcal{O}_{x} \rightarrow 0
$$

Si $x \in \operatorname{supp} G$ alors $\underline{\operatorname{Tor}}_{1}\left(G, \mathcal{O}_{x}\right) \neq 0$ (voir la démonstration du lemme 5.2) et donc $h^{0}\left(F^{\prime} \otimes G\right) \neq 0$. Sinon $h^{0}\left(F^{\prime} \otimes G\right)=h^{0}(F \otimes G)$. On déduit que $\sigma_{F^{\prime}}=\operatorname{cst} \cdot \sigma_{F} \cdot H_{x}$.

Preuve du lemme 5.7. - Pour $a, b \in \mathbb{P}_{2}$, la classe du faisceau $F=I_{a} \oplus I_{b}$ appartient à $\mathrm{M}_{\left(2,0, c_{2}=2\right)}$. On commence par prouver que $\sigma_{F}=\operatorname{cst} \cdot \theta^{2} \cdot \pi^{*}\left(H_{a} \cdot H_{b}\right)$. La section $\sigma_{F}$ s'annule sur $\pi^{-1}\left(\left\{H_{a}=0\right\}\right)$ et sur $\pi^{-1}\left(\left\{H_{b}=0\right\}\right)$ d'après le lemme 5.2. Alors la section rationnelle $\alpha=\sigma_{F} / \pi^{*}\left(H_{a} \cdot H_{b}\right)$ de $\Theta^{\otimes 2}$ est régulière. $\mathrm{Si} d=1,2$ on applique la proposition 4.4 pour avoir un isomorphisme $\mathrm{H}^{0}\left(C_{d}, \mathcal{O}\right)=\mathrm{H}^{0}\left(\mathrm{M}_{d \mathfrak{u}}, \Theta^{\otimes 2}\right)$. Pour $d=3$ cet isomorphisme découle du corollaire 4.13 appliqué pour $n=0$. Par conséquent $\alpha=\operatorname{cst} \cdot \theta^{2}$.

Puisque les produits $H_{a} \cdot H_{b}$ engendrent $\mathrm{H}^{0}\left(C_{d}, \mathcal{O}(2)\right)$, le lemme 5.7 est prouvé pour $d=1,2$.

Pour $d=3$, en regardant la suite exacte du corollaire 4.13, on montre que les $\sigma_{F}$ engendrent le sous-espace $\theta^{2} \cdot \pi^{*} \mathrm{H}^{0}\left(C_{3}, \mathcal{O}(2)\right)$ de $\mathrm{H}^{0}\left(\mathrm{M}_{d \mathfrak{u}}, \Theta^{\otimes 2}(2)\right)$. Pour montrer le lemme, en tenant compte du fait que $h^{0}\left(C_{3}, \mathcal{O}\right)=1$, il suffit de trouver un faisceau $F$ pour lequel $s^{*} \sigma_{F} \neq 0$. Ceci équivaut à trouver une cubique $C$ pour laquelle $h^{0}\left(F_{\mid C}\right)=h^{1}\left(F_{\mid C}\right)=0$. Cette condition est satisfaite pour tout faisceau $F$ localement libre stable et toute cubique $C$. Effectivement, si on reconsidère la résolution de $\mathcal{O}_{C}$

$$
0 \rightarrow \mathcal{O}(-3) \stackrel{\cdot \underline{C}}{\longrightarrow} \mathcal{O} \longrightarrow \mathcal{O}_{C} \rightarrow 0,
$$

BULlETIN DE LA SOCIÉTÉ MATHÉMATIQUE DE FRANCE 
comme $\underline{\operatorname{Tor}}_{1}\left(F, \mathcal{O}_{C}\right)=0$, on obtient

$$
0 \rightarrow F(-3) \stackrel{\cdot \underline{C}}{\longrightarrow} F \longrightarrow F_{\mid C} \rightarrow 0 .
$$

Alors la suite

$$
\mathrm{H}^{1}(F) \longrightarrow \mathrm{H}^{1}\left(F_{\mid C}\right) \rightarrow \mathrm{H}^{2}(F(-3))
$$

est exacte. Le nombre de Hodge $h^{1}(F)=n-2$ est nul pour $n=2$ et par la dualité de Serre on a $\mathrm{H}^{2}(F(-3))=\mathrm{H}^{0}\left(F^{*}\right)=\operatorname{Hom}(F, \mathcal{O})$. Ce dernier groupe est nul, d'où $h^{1}\left(F_{\mid C}\right)=0$. L'annulation de $\operatorname{Hom}(F, \mathcal{O})$ s'obtient ainsi : s'il existe un morphisme non nul $m: F \rightarrow \mathcal{O}$, alors $\operatorname{im} m \subset \mathcal{O}$, donc im $m=I_{Z}$, pour $Z$ sous-schéma dans $\mathbb{P}_{2}$. La stabilité de $F$ implique $c_{1}\left(I_{Z}\right)=0$ et $\chi\left(I_{Z}\right)>0$, soit que $I_{Z}=\mathcal{O}$. Alors Ker $m$ est localement libre de $c_{1}=0$ et $\chi=-1$, ce qui est contradictoire. Donc $\operatorname{Hom}(F, \mathcal{O})=0$.

\section{Preuve de la proposition 1.2}

On reprend la démarche et les notations de l'article [5]. Dans cet article, on se fixait un entier positif $\ell$, qui dans cette application sera toujours égal à 1 . On introduit la notion de système cohérent, qui consiste à considérer en même temps que le faisceau $F$, un sous-espace vectoriel $\Gamma$ de son espace de sections $\mathrm{H}^{0}(F)$. La dimension de $\Gamma$ donne l'ordre du système cohérent. À l'aide de résultats de Min He [13] sur les espaces de modules de systèmes cohérents $(\Gamma, F(\ell))$ d'ordre 1, dont le faisceau sous-jacent est de rang 2 , et de classes de Chern $c_{1}=2 \ell, c_{2}=n+\ell^{2}$, on se ramène au paragraphe 3 de [5], pour $n$ compris entre $\ell(\ell-1)$ et $(\ell+1)(\ell+2)$, à l'étude de l'espace des sections d'un fibré vectoriel $\mathbf{S}^{\ell d} \mathcal{R} \otimes \mathfrak{d}^{\otimes d}$ sur un ouvert $U$ du schéma de Hilbert $\operatorname{Hilb}^{m}\left(\mathbb{P}_{2}\right)$ des sous-schémas finis de longueur $m=n+\ell^{2}$.

$\operatorname{Si} \Xi \subset \operatorname{Hilb}^{m}\left(\mathbb{P}_{2}\right) \times \mathbb{P}_{2}$ est le sous-schéma universel, $\mathcal{I}$ est le faisceaux d'idéaux associé,

$$
\operatorname{pr}_{1}: \operatorname{Hilb}^{m}\left(\mathbb{P}_{2}\right) \times \mathbb{P}_{2} \longrightarrow \operatorname{Hilb}^{m}\left(\mathbb{P}_{2}\right), \quad \operatorname{pr}_{2}: \operatorname{Hilb}^{m}\left(\mathbb{P}_{2}\right) \times \mathbb{P}_{2} \longrightarrow \mathbb{P}_{2}
$$

sont les deux projections, le faisceau algébrique cohérent $\mathcal{R}$ est défini par

$$
\mathcal{R}=R^{1} \operatorname{pr}_{1 *}(\mathcal{I}(2 \ell-3)) .
$$

Ce faisceau est localement libre en dehors du fermé de Brill-Noether $B$ des schémas $Z \in \operatorname{Hilb}^{m}\left(\mathbb{P}_{2}\right)$ tels que $h^{0}\left(I_{Z}(2 \ell-3)\right) \neq 0$. On note $U$ l'ouvert complémentaire de $B$. La codimension de $B$ est supérieure ou égale à 2 , donc les résultats de cohomologie locale nous permettent de passer de $\operatorname{Hilb}^{m}\left(\mathbb{P}_{2}\right)$ à $U$ pour le calcul d'un espace de sections.

Le fibré $\mathfrak{d}=\mathcal{D}_{\mathfrak{u}}$ est le fibré déterminant sur le schéma de Hilbert $\operatorname{Hilb}^{m}\left(\mathbb{P}_{2}\right)$, identifié à l'espace de modules $\mathrm{M}_{\left(1,0, c_{2}=n\right)}$ comme dans le lemme 5.5. L'énoncé précis démontré dans [5] est :

TOME $130-2002-\mathrm{N}^{\mathrm{O}} 1$ 
THÉORÈme 6.1. - Soit $n$ un entier $\geq 3$. Soit $\ell$ un entier $>0$ tel que l'on ait $\ell(\ell-1) \leq n<(\ell+1)(\ell+2)$. Alors on a un isomorphisme de SL(3)-représentations

$$
\mathrm{H}^{0}\left(\mathrm{M}_{c}, \mathcal{D}^{\otimes d}\right)=\mathrm{H}^{0}\left(U, \mathbf{S}^{\ell d} \mathcal{R} \otimes \mathfrak{d}^{\otimes d}\right) .
$$

On désigne par $E$ l'espace de sections $\mathrm{H}^{0}\left(\mathbb{P}_{2}, \mathcal{O}(1)\right)$. Au paragraphe 4 de [5] on montre que $\mathbf{S}^{l d} \mathcal{R} \otimes \mathfrak{d}^{\otimes d}$ admet sur $U$ une résolution $\left(^{*}\right)$ par un complexe

$$
K^{-i}=\Lambda^{i} \mathbf{S}^{k} E \otimes \mathbf{S}^{\ell d-i}\left(\mathcal{O}(k)^{[m]}\right) \otimes \mathfrak{d}^{\otimes d}
$$

pour $i=0, \ldots, l d$, où $k=2 \ell-3$, et $\mathcal{O}(k)^{[m]}$ est défini par

$$
\mathcal{O}(k)^{[m]}=\operatorname{pr}_{1 *}\left(\mathcal{O}_{\Xi} \otimes \operatorname{pr}_{2}^{*}(\mathcal{O}(k))\right) .
$$

Il est prouvé dans [5] le théorème suivant :

ThÉORÈme 6.2. - On a sur $\operatorname{Hilb}^{m}\left(\mathbb{P}_{2}\right)$ :

(i) $\mathrm{H}^{0}\left(\mathfrak{d}^{\otimes d}\right)=\mathbf{S}^{m}\left(\mathbf{S}^{d} E\right)$;

(ii) $\mathrm{H}^{0}\left(\mathcal{O}(k)^{[m]} \otimes \mathfrak{d}^{\otimes d}\right)=\mathbf{S}^{k+d} E \otimes \mathbf{S}^{m-1}\left(\mathbf{S}^{d} E\right)$;

(iii) la $\mathrm{SL}(3)$-représentation $\mathrm{H}^{0}\left(\mathbf{S}^{2}\left(\mathcal{O}(k)^{[m]}\right) \otimes \mathfrak{d}^{\otimes d}\right)$ est isomorphe à la représentation $\left(\mathbf{S}^{2 k+d} E \otimes \mathbf{S}^{m-1}\left(\mathbf{S}^{d} E\right)\right) \oplus\left(\operatorname{Ker}_{k} \otimes \mathbf{S}^{m-2}\left(\mathbf{S}^{d} E\right)\right)$ où $\operatorname{Ker}_{k}$ est le noyau de la multiplication $\mathbf{S}^{2}\left(\mathbf{S}^{k+d} E\right) \rightarrow \mathbf{S}^{2 k+2 d} E$;

(iv) la $\mathrm{SL}(3)$-représentation $\mathrm{H}^{0}\left(\mathbf{S}^{3}\left(\mathcal{O}(k)^{[m]}\right) \otimes \mathfrak{d}^{\otimes d}\right)$ est le noyau du morphisme

$$
\begin{array}{r}
\alpha:\left[\mathbf{S}^{3 k+d} E \otimes \mathbf{S}^{m-1}\left(\mathbf{S}^{d} E\right)\right] \oplus\left[\mathbf{S}^{2 k+d} E \otimes \mathbf{S}^{k+d} E \otimes \mathbf{S}^{m-2}\left(\mathbf{S}^{d} E\right)\right] \\
\oplus\left[\mathbf{S}^{3}\left(\mathbf{S}^{k+d} E\right) \otimes \mathbf{S}^{m-3}\left(\mathbf{S}^{d} E\right)\right] \\
\longrightarrow\left[\mathbf{S}^{3 k+2 d-1} E \otimes E \otimes \mathbf{S}^{m-2}\left(\mathbf{S}^{d} E\right)\right] \\
\oplus\left[\mathbf{S}^{2 k+2 d} E \otimes \mathbf{S}^{k+d} E \otimes \mathbf{S}^{m-3}\left(\mathbf{S}^{d} E\right)\right]
\end{array}
$$

donné par la matrice

$$
\left(\begin{array}{ccc}
\widetilde{\nabla} & \widetilde{D} & 0 \\
0 & \rho & \widetilde{\nu}
\end{array}\right)
$$

où $\widetilde{\nabla}, \widetilde{D}, \rho$ et $\widetilde{\nu}$ sont des opérateurs explicites.

Le point (i) est expliqué après le lemme 5.5. Le point (ii) correspond au lemme 4.10 de [5]. Le point (iii) correspond au lemme 4.11 de [5]. Le point (iv) correspond à la proposition 5.13 de [5].

Le théorème 6.1 appliqué au cas $\ell=1$ nous donne un isomorphisme de SL(3)-représentations

$$
\mathrm{H}^{0}\left(\mathrm{M}_{c}, \mathcal{D}^{\otimes d}\right)=\mathrm{H}^{0}\left(\mathrm{Hilb}^{n+1} \mathbb{P}_{2}, \mathbf{S}^{d} \mathcal{R} \otimes \mathfrak{d}^{\otimes d}\right) \text { pour } 3 \leq n \leq 5 .
$$

BULLETIN DE LA SOCIÉTÉ MATHÉMATIQUE DE FRANCE 
À partir de la présentation $\left(^{*}\right)$ de $\mathcal{R}$, appliquée pour $k=2 \ell-3=-1$ et $m=n+\ell^{2}=n+1$, on obtient $\mathcal{O}(-1)^{[m]} \simeq \mathcal{R}$ et donc

$$
\mathrm{H}^{0}\left(\mathrm{M}_{c}, \mathcal{D}^{\otimes d}\right)=\mathrm{H}^{0}\left(\mathrm{Hilb}^{n+1} \mathbb{P}_{2}, \mathbf{S}^{d}\left(\mathcal{O}(-1)^{[m]}\right) \otimes \mathfrak{d}^{\otimes d}\right) \quad \text { pour } 3 \leq n \leq 5 .
$$

Pour $d=2$ on applique le théorème 6.2 (iii) avec $k=2 \ell-3=-1, d=2$ et $m=n+\ell^{2}=n+1: \mathrm{H}^{0}\left(\mathrm{M}_{c}, \mathcal{D}^{\otimes 2}\right)$ est le noyau du morphisme surjectif

$$
\mathbf{S}^{n}\left(\mathbf{S}^{2} E\right) \oplus \mathbf{S}^{2} E \otimes \mathbf{S}^{n-1}\left(\mathbf{S}^{2} E\right) \stackrel{(0, \text { id })}{\longrightarrow} \mathbf{S}^{2} E \otimes \mathbf{S}^{n-1}\left(\mathbf{S}^{2} E\right) .
$$

Par suite, la représentation $\mathrm{H}^{0}\left(\mathrm{M}_{c}, \mathcal{D}^{\otimes 2}\right)$ est isomorphe à $\mathbf{S}^{n}\left(\mathbf{S}^{2} E\right)$, de dimension $C_{n+5}^{n}$.

Pour $d=3$ on s'intéresse à l'espace $\mathrm{H}^{0}\left(\mathrm{Hilb}^{n+1} \mathbb{P}_{2}, \mathbf{S}^{3}\left(\mathcal{O}(-1)^{[m]}\right) \otimes \mathfrak{d}^{\otimes 3}\right)$. Par le théorème 6.2 (iv), on est amenés à étudier le noyau du morphisme $\alpha$ (on fait $k=-1, d=3$ et $m=n+1)$ :

$$
\begin{aligned}
\alpha: \mathbf{S}^{n}\left(\mathbf{S}^{3} E\right) & \oplus E \otimes \mathbf{S}^{2} E \otimes \mathbf{S}^{n-1}\left(\mathbf{S}^{3} E\right) \oplus \mathbf{S}^{3}\left(\mathbf{S}^{2} E\right) \otimes \mathbf{S}^{n-2}\left(\mathbf{S}^{3} E\right) \\
& \rightarrow E \otimes \mathbf{S}^{2} E \otimes \mathbf{S}^{n-1}\left(\mathbf{S}^{3} E\right) \oplus \mathbf{S}^{4} E \otimes \mathbf{S}^{2} E \otimes \mathbf{S}^{n-2}\left(\mathbf{S}^{3} E\right) .
\end{aligned}
$$

On montre successivement selon une démarche analogue à celle des lemmes $5.16,5.17,5.19,5.20$ de l'article [5], que $\widetilde{D}$ est un isomorphisme, que le noyau de $\widetilde{\nu}$ est égal à $\mathbf{S}^{2,2,2} E \otimes \mathbf{S}^{n-2}\left(\mathbf{S}^{3} E\right)=\mathbb{C} \otimes \mathbf{S}^{n-2}\left(\mathbf{S}^{3} E\right)$ et son conoyau égal à $\mathbf{S}^{5,1} E \otimes \mathbf{S}^{n-2}\left(\mathbf{S}^{3} E\right)$, et que le morphisme de liaison

$$
(0, \rho): \operatorname{Ker}(\widetilde{\nabla}, \widetilde{D}) \longrightarrow \operatorname{coker} \widetilde{\nu}
$$

est nul. Par conséquent, l'équivalent de la proposition 5.18 de [5] nous assure que le noyau de $\alpha$ est isomorphe à $\mathbf{S}^{n}\left(\mathbf{S}^{3} E\right) \oplus \mathbf{S}^{n-2}\left(\mathbf{S}^{3} E\right)$, de dimension $C_{n+9}^{9}+C_{n+7}^{9}$.

Ceci conclut la preuve du théorème 1.1.

\section{Sections de $\mathcal{D}^{\otimes k}$ pour $n=c_{2} \leq 4$}

Le but de ce paragraphe est de calculer les dimensions des espaces de sections $\mathrm{H}^{0}\left(\mathrm{M}_{\left(2,0, c_{2}=n\right)}, \mathcal{D}_{\mathfrak{u}}{ }^{\otimes k}\right)$ pour $n \leq 4$.

Dans le cas $n=2$, le morphisme de Barth nous fournit un isomorphisme entre l'espace de modules $\mathrm{M}_{c}$ et $\mathbb{P}_{5}$, et le fibré déterminant s'identifie à $\mathcal{O}(1)$. Les cas intéressants sont donc $n=3$ et $n=4$.

On commence par un résultat général sur la fonction $k \mapsto h^{0}\left(\mathrm{M}_{c}, \mathcal{D}_{u}^{\otimes k}\right)$ pour toute classe $c=\left(r, c_{1}, c_{2}\right)$ satisfaisant $r>0$ et $\mathrm{M}_{c}$ non-vide. On rappelle ( $(3.2)$ la notation $\mathfrak{u}=\left(0, r / \delta,-c_{1} / \delta\right)$ où $\delta=\operatorname{pgcd}\left(\mathrm{r}, \mathrm{c}_{1}\right)$. D'après le théorème 3.8, la cohomologie supérieure $\mathrm{H}^{q}\left(\mathrm{M}_{c}, \mathcal{D}_{\mathfrak{u}}{ }^{\otimes k}\right)$ s'annule pour $k \geq-3 \delta$.

TOME $130-2002-\mathrm{N}^{\mathrm{O}} 1$ 
Ce résultat, l'isomorphisme $\omega_{\mathrm{M}_{c}} \simeq \mathcal{D}_{\mathfrak{u}}{ }^{\otimes-3 \delta}$ et la dualité de Serre nous assurent que

$$
\begin{aligned}
& h^{0}\left(\mathcal{D}^{\otimes j}\right)=0 \quad \text { si } j<0, \\
& h^{q}\left(\mathcal{D}^{\otimes j}\right)=0 \text { pour tout } j \text { si } 0<q<D, \\
& h^{D}\left(\mathcal{D}^{\otimes j}\right)=0 \text { si } j>-3 \delta \text {. }
\end{aligned}
$$

On note $D=\operatorname{dim} \mathrm{M}_{c}=1-\left\langle c, c^{*}\right\rangle$. On note $\mathcal{D}=\mathcal{D}_{\mathfrak{u}}$.

Proposition 7.1. - (i) Supposons $d \geq 2$. Pour $k>-3 \delta$, la fonction

$$
k \longmapsto h^{0}\left(\mathrm{M}_{c}, \mathcal{D}^{\otimes k}\right)
$$

est un polynôme de degré $D$, de coefficient dominant

$$
q_{D}=\frac{1}{D !} \int_{\mathrm{M}_{c}} c_{1}(\mathcal{D})^{D} .
$$

(ii) La série de Poincaré $P(t)$ est de la forme

$$
\frac{Q(t)}{(1-t)^{D+1}}
$$

où $Q$ est un polynôme de degré $D+1-3 \delta$, à coefficients entiers, tel que $Q(1)=\int_{\mathrm{M}_{c}} c_{1}(\mathcal{D})^{D}$.

(iii) Le polynôme $Q$ satisfait à la condition de symétrie

$$
t^{D+1-3 \delta} Q\left(\frac{1}{t}\right)=Q(t) .
$$

Preuve. - La formule de Riemann-Roch pour des variétés éventuellement singulières (voir [3]) et le théorème 3.8 donnent

$$
\begin{aligned}
h^{0}\left(\mathrm{M}_{c}, \mathcal{D}^{\otimes k}\right) & =\chi\left(\mathrm{M}_{c}, \mathcal{D}^{\otimes k}\right)=\int_{\mathrm{M}_{c}} \operatorname{ch}\left(\mathcal{D}^{\otimes k}\right) \operatorname{Td}\left(\mathrm{M}_{c}\right) \\
& =\int_{\mathrm{M}_{c}} \mathrm{e}^{k c_{1}(\mathcal{D})} \operatorname{Td}\left(\mathrm{M}_{c}\right)=\sum_{0 \leq j \leq D} \frac{k^{j}}{j !} \int_{\mathrm{M}_{c}} c_{1}(\mathcal{D})^{j} \operatorname{Td}\left(\mathrm{M}_{c}\right) .
\end{aligned}
$$

Puisque $\mathcal{D}$ est big $q_{D}>0$, d'où (i).

Posons $a_{j}=\int_{\mathrm{M}_{c}} c_{1}(\mathcal{D})^{j} \operatorname{Td}\left(\mathrm{M}_{c}\right)$. La série de Poincaré est

$$
\sum_{0 \leq j \leq D} a_{j}\left(\sum_{k \geq 0} \frac{k^{j}}{j !} t^{k}\right)
$$

La somme de la série $\sum_{k \geq 0} k^{j} / j ! t^{k}$ (de rayon de convergence 1) est une fonction rationnelle de la forme $Q_{j}(t) /(1-t)^{j+1}$ où les polynômes $Q_{j}$ sont de degré inférieur où égal à $j$, et $Q_{j}(1)=1$. Ceci se voit par récurrence sur $j$. Il en résulte que la série de Poincaré est de la forme voulue. Puisque $Q(t)=(1-t)^{D+1} P(t)$

BULLETIN DE LA SOCIÉTÉ MATHÉMATIQUE DE FRANCE 
et que $P(t)$ est une série formelle à coefficients entiers, le calcul de ce produit montre que $Q(t)$ est bien à coefficients entiers.

La relation classique (voir par exemple [10])

$$
\operatorname{Td}\left(V^{*}\right) \operatorname{ch}\left(\lambda_{-1}(V)\right)=c_{\text {top }}\left(V^{*}\right)
$$

appliquée au fibré $V=W \otimes \mathcal{D}^{-1}$, où $W$ est un espace vectoriel de dimension $m=D+1$, prouve que

$$
\operatorname{ch}\left(\lambda_{-1}\left(W \otimes \mathcal{D}^{-1}\right)\right)=c_{\text {top }}\left(W^{*} \otimes \mathcal{D}\right) \operatorname{Td}^{-1}\left(W^{*} \otimes \mathcal{D}\right) .
$$

Ici

$$
\lambda_{-1}(V)=\Lambda^{0} V-\Lambda^{1} V+\Lambda^{2} V-\cdots+(-1)^{\operatorname{dim} V} \Lambda^{\operatorname{dim} V} V
$$

désigne la somme alternée des puissances extérieures du fibré $V$ et $c_{\text {top }}$ d'un fibré vectoriel de rang $r$ désigne la classe de Chern $c_{r}$ de ce fibré.

Mais $V=W \otimes \mathcal{D}^{-1}$ est un fibré de rang $D+1$, donc sa classe de Chern maximale $c_{D+1}\left(W \otimes \mathcal{D}^{-1}\right)$ appartient à l'espace de cohomologie $\mathrm{H}^{2(D+1)}\left(\mathrm{M}_{c}\right)$ qui est nul en raison de la dimension de $\mathrm{M}_{c}\left(\operatorname{dim} \mathrm{M}_{c}=D\right)$. Donc

$$
\operatorname{ch}\left(\lambda_{-1}\left(W \otimes \mathcal{D}^{-1}\right) \otimes \mathcal{D}^{\otimes k}\right)=0
$$

et par la formule de Riemann-Roch on obtient

$$
\chi\left(\lambda_{-1}\left(W \otimes \mathcal{D}^{-1}\right) \otimes \mathcal{D}^{\otimes k}\right)=\int_{\mathrm{M}_{c}} \operatorname{ch}\left(\lambda_{-1}\left(W \otimes \mathcal{D}^{-1}\right) \otimes \mathcal{D}^{\otimes k}\right) \operatorname{Td}\left(\mathrm{M}_{c}\right)=0
$$

soit, en tenant compte de $\Lambda^{i}\left(W \otimes \mathcal{D}^{-1}\right)=\Lambda^{i} W \otimes \mathcal{D}^{\otimes-i}$,

$$
S_{k}=\sum_{i=0}^{m}(-1)^{i} C_{m}^{i} \chi\left(\mathcal{D}^{\otimes k-i}\right)=0
$$

quelque soit $k$.

Comme $Q(t)=(1-t)^{D+1} P(t)$, le coefficient $k$-ième de $Q$ s'écrit

$$
\begin{aligned}
Q_{k} & =\left(\begin{array}{c}
m \\
0
\end{array}\right) h^{0}\left(\mathcal{D}^{\otimes k}\right)-\left(\begin{array}{c}
m \\
1
\end{array}\right) h^{0}\left(\mathcal{D}^{\otimes k-1}\right)+\cdots+(-1)^{m}\left(\begin{array}{c}
m \\
m
\end{array}\right) h^{0}\left(\mathcal{D}^{\otimes k-m}\right) \\
& =\sum_{i=0}^{m}(-i)^{i} C_{m}^{i} h^{0}\left(\mathcal{D}^{\otimes k-i}\right)
\end{aligned}
$$

(en tenant compte du fait que $h^{0}\left(\mathcal{D}^{\otimes j}\right)=0$ si $j<0$ ). Pour $k<m-3 \delta$, on obtient $Q_{k}=S_{k}=0$ en raison de l'annulation de la cohomologie supérieure $\mathrm{H}^{q}\left(\mathcal{D}^{\otimes j}\right)$ pour $j<-3 \delta, q>0$ et de $\mathrm{H}^{0}\left(\mathcal{D}^{\otimes j}\right)$ pour $j<0$. D'où deg $Q \leq m-3 \delta$. La condition de symétrie s'exprime sur la symétrie des coefficients de $Q$ :

$$
Q_{k}=Q_{m-3 \delta-k} \text { pour } \quad 0 \leq k \leq m-3 \delta .
$$

Mais dans ce cas on a

$$
Q_{k}=S_{k}-\sum_{i=k+3 \delta}^{m}(-i)^{i}\left(\begin{array}{c}
m \\
i
\end{array}\right) \chi\left(\mathcal{D}^{\otimes k-i}\right) .
$$

TOME $130-2002-\mathrm{N}^{\mathrm{O}} 1$ 
La dualité de Serre s'écrit pour $j \leq-3 \delta$ sous la forme

$$
\chi\left(\mathcal{D}^{\otimes j}\right)=(-1)^{D} h^{0}\left(\mathcal{D}^{\otimes-j-3 \delta}\right) .
$$

En l'appliquant dans la somme ci-dessus pour $j=k-i \leq-3 \delta$, et en faisant ensuite la transformation $j=m-i$ on trouve

$$
\begin{aligned}
Q_{k} & =S_{k}-(-1)^{m} \sum_{i=k+3 \delta}^{m}(-i)^{m-i}\left(\begin{array}{c}
m \\
m-i
\end{array}\right)(-1)^{D} h^{0}\left(\mathcal{D}^{\otimes-k+i-3 \delta}\right) \\
& =S_{k}+\sum_{j=0}^{m-k-3 \delta}(-i)^{j}\left(\begin{array}{c}
m \\
j
\end{array}\right) h^{0}\left(\mathcal{D}^{\otimes m-k-3 \delta-j}\right) \\
& =S_{k}+Q_{m-3 \delta-k}=Q_{m-3 \delta-k} .
\end{aligned}
$$

En particulier $Q_{0}=Q_{m-3 \delta}=1$ donc le degré de $Q$ est égal à $m-3 \delta$ exactement.

Nous revenons aux cas particuliers qui nous intéressent. On prend $c=$ $\left(2,0, c_{2}=n\right)$ pour $n=3,4, \delta=2$ et $\mathfrak{u}=(0,1,0)$.

Preuve du théorème 1.3. - (i) Ici, $D=4 c_{2}-3=9$. La proposition précédente donne que $P(t)$ s'écrit sous la forme $Q(t) /(1-t)^{10}$ où $Q$ est de degré 4 , vérifie la condition de symétrie et $Q(1)$ est égal à $3=9 ! q_{9}$ où $q_{9}$ est un nombre de Donaldson (voir [2]). Le calcul de $h^{0}\left(\mathrm{M}_{c}, \mathcal{D}^{0}\right)=1$ et $h^{0}\left(\mathrm{M}_{c}, \mathcal{D}\right)=10(c f .[5])$ permet de conclure que $Q(t)=1+t^{2}+t^{4}$.

(ii) Ce cas est analogue au précédent; seulement il faut faire intervenir $h^{0}\left(\mathrm{M}_{c}, \mathcal{D}\right)=15(c f .[5])$, et aussi $h^{0}\left(\mathrm{M}_{c}, \mathcal{D}^{\otimes 2}\right)=126$ et $h^{0}\left(\mathrm{M}_{c}, \mathcal{D}^{\otimes 3}\right)=770$ calculés au $\S 1.2$. Ici $Q(1)=54=13$ ! $q_{13}$ (voir [22]).

Remarque 7.2. - Dans le cas $n=3$, puisqu'on a obtenu $h^{0}\left(\mathrm{M}_{c}, \mathcal{D}^{\otimes d}\right)$ pour $d=2,3$ ( $c f .1 .2$ ), on aurait pu déduire la valeur du nombre de Donaldson $q_{9}$.

Remerciements. - Mes remerciements s'adressent à J. Le Potier, mon directeur de thèse, ainsi qu'à N. Dan. Je remercie D. Roessler pour la référence [10].

\section{BIBLIOGRAPHIE}

[1] Altman (A.), Iarrobino (A.) \& Kleiman (S.) - Irreducibility of the compactified jacobian, in Nordic Summer School/NAVF, Symposium in Mathematics, Oslo, August 5-25, 1976.

[2] BARTH (W.) - Moduli of vector bundles on projective plane, Invent. Math., t. 42 (1977), pp. 63-91.

[3] Baum (P.), Fulton (W.) \& MacPherson (R.) - Riemann-Roch and topological $\mathcal{K}$ theory for singular varieties, Acta Math., t. 143 (1979), no. 34, pp. 155-192.

BULletin DE LA SOCIÉtÉ MATHÉMATIQUE DE FRANCE 
[4] Boutot (J.-F.) - Singularités rationnelles et quotients par les groupes réductifs, Invent. Math., t. 88 (1987), pp. 65-68.

[5] Danila (G.) - Sections du fibré déterminant sur l'espace de modules des faisceaux semi-stables de rang 2 sur le plan projectif, Ann. Inst. Fourier Grenoble, t. 50 (2000), pp. 1323-1374.

[6] DrÉZet (J.-M.) - Fibrés exceptionnels et variétés de modules de faisceaux semi-stables sur $\mathbb{P}_{2}$, J. reine angew. Math., t. 380 (1987), pp. 14-58.

[7] _ Groupe de Picard des variétés de modules de faisceaux semistables sur $\mathbb{P}_{2}(\mathbb{C})$, Ann. Inst. Fourier Grenoble, t. 38 (1988), pp. 105-168.

[8] Drézet (J.-M.) \& Narasimhan (M.S.) - Groupe de Picard des variétés de modules de fibrés semi-stables sur les courbes algébriques, Invent. Math., t. 97 (1989), pp. 53-94.

[9] Esnault (H.) \& Viehweg (E.) - Lectures on vanishing theorems, DMV Seminar, vol. 20, Birkhäuser Verlag, Basel, 1992.

[10] Fulton (W.) - Intersection theory, Springer-Verlag, 1984.

[11] Grothendieck (A.) - Local cohomology, Lecture Notes Series, vol. 41, 1967.

[12] Hartshorne (R.) - Algebraic Geometry, Graduate Texts in Mathematics, vol. 52, Springer-Verlag, 1977.

[13] He (Min) - Espaces de modules de systèmes cohérents, Int. J. of Maths, t. 9-5 (1998), pp. 545-598.

[14] Le Potier (J.) - Dualité étrange sur le plan projectif, exposé donné à Luminy en décembre 1996.

[15] L L'espace de modules de Simpson, in Séminaire de géométrie algébrique, Jussieu, exposé du 27 février 1992.

[16] _ Fibré déterminant et courbes de saut sur les surfaces algébriques, in Complex projective Geometry, Lecture Notes Series, vol.179, London Math. Soc., 1992, pp. 213-240.

[17] _ Faisceaux semi-stables de dimension 1 sur le plan projectif, Revue roumaine de math. pures et appliquées, t. 38 (1993), pp. 635-678, dédicacé à la mémoire de Constantin Banica.

[18] _ Faisceaux semi-stables et systèmes cohérents, in Proceedings de la Conference de Durham (juillet 1993), Cambridge University Press, 1995, pp. 179-239.

[19] _ Module des fibrés semi-stables et fonctions thêta, in Proceedings du Symposium Taniguchi sur les fibrés vectoriels (Kyoto, décembre 1994); Moduli of vector bundles (Maruyama (M.), éd.), Lecture Notes in Pure and Applied Math., vol. 179, 1996, pp. 83-101.

[20] _ Lectures on vector bundles, Cambridge Studies in Advanced Mathematics, vol. 54, Cambridge Univ. Press, 1997.

[21] Li (JUN) - Algebraic Geometric Interpretation of Donaldson's Polynomial Invariants, J. Diff. Geo., t. 37 (1993), pp. 417-466.

TOME $130-2002-\mathrm{N}^{\mathrm{O}} 1$ 
[22] Li (W.-P.) \& QIN (Z.) - Lower-degree Donaldson Polynomial Invariants of Rational Surfaces, J. Alg. Geom., t. 2 (1993), pp. 413-442.

[23] Milne (J.S.) - Abelian Varieties, in Arithmetic Geometry (Cornell (G.) \& Silverman (J.H.), éds.), Springer-Verlag, 1986.

[24] Mumford (D.) \& Fogarty (J.) - Geometric Invariant Theory, 2nd ed., Ergebnisse der Mathematik und ihrer Grenzgebiete, vol. 34, SpringerVerlag, 1982.

[25] O'Grady (K.G.) - Moduli of vector bundles on surfaces, in Algebraic geometry - Santa Cruz 1995, Proc. Sympos. Pure Math., Part 1, vol. 62, Amer. Math. Soc., Providence, RI, 1997, pp. 101-126.

[26] Simpson (C.T.) - Moduli of Representations of the Fundamental Group of a Smooth Variety, Publ. Math. IHES, t. 79-80 (1994). 